\title{
Avoiding Communication in Successive Band Reduction
}

\author{
GREY BALLARD, University of California, Berkeley and Sandia National Laboratories \\ JAMES DEMMEL and NICHOLAS KNIGHT, University of California, Berkeley
}

The running time of an algorithm depends on both arithmetic and communication (i.e., data movement) costs, and the relative costs of communication are growing over time. In this work, we present sequential and distributed-memory parallel algorithms for tridiagonalizing full symmetric and symmetric band matrices that asymptotically reduce communication compared to previous approaches.

The tridiagonalization of a symmetric band matrix is a key kernel in solving the symmetric eigenvalue problem for both full and band matrices. In order to preserve structure, tridiagonalization routines use annihilate-and-chase procedures that previously have suffered from poor data locality and high parallel latency cost. We improve both by reorganizing the computation and obtain asymptotic improvements. We also propose new algorithms for reducing a full symmetric matrix to band form in a communication-efficient manner. In this article, we consider the cases of computing eigenvalues only and of computing eigenvalues and all eigenvectors.

Categories and Subject Descriptors: G.1.3 [Numerical Analysis]: Numerical Linear Algebra-Eigenvalues and eigenvectors (direct and iterative methods); F.2.1 [Analysis of Algorithms and Problem Complexity]: Numerical Algorithms and Problems-Computations on matrices

General Terms: Algorithms, Performance, Theory

Additional Key Words and Phrases: Symmetric eigenvalue problem, communication avoiding algorithms, band reduction

\section{ACM Reference Format:}

Ballard, G., Demmel, J., and Knight, N. 2013. Avoiding communication in successive band reduction. ACM Trans. Parallel Comput. 1, 2, Article 11 (January 2015), 37 pages.

DOI : http://dx.doi.org/10.1145/2686877

\section{INTRODUCTION}

The running time of an algorithm is a function of both the number of arithmetic operations performed and the amount of data moved through the memory hierarchy of a single processor and, in the distributed-memory parallel case, across a network between processors. The communication costs, that is, the costs of moving data, on today's machines often greatly exceed the cost of performing arithmetic operations on it, and architectural trends indicate that this processor-memory gap is growing exponentially over time [Fuller and Millett 2011]. Thus, we are interested in new algorithms which

The authors acknowledge funding from Microsoft (award \#024263) and Intel (award \#024894), and matching funding by UC Discovery (award \#DlG07-10227), with additional support from ParLab affiliates National Instruments, Nokia, NVIDIA, Oracle, and Samsung; from DARPA (award \#HR0011-12-2-0016) and the Center for Future Architecture Research, a member of STARnet, a Semiconductor Research Corporation program sponsored by MARCO and DARPA; from Math Works; and from the US DOE (grants DE-SC0003959, DESC0004938, DE-SC0005136, DE-SC0010200, DESC0008700, and DE-AC02-05CH11231 and NSF (grants ACl-1339676 and OCl-1032639). This research was supported in part by an appointment to the Sandia National Laboratories Truman Fellowship in National Security Science and Engineering, sponsored by Sandia Corporation (a wholly owned subsidiary of Lockheed Martin Corporation) as Operator of Sandia National Laboratories under its U.S. Department of Energy Contract No. DE-AC04-94AL85000.

Author's address: G. Ballard (corresponding author); email: gmballa@sandia.gov.

(c) 2015 Association for Computing Machinery. ACM acknowledges that this contribution was authored or co-authored by an employee, contractor or affiliate of the United States government. As such, the United States Government retains a nonexclusive, royalty-free right to publish or reproduce this article, or to allow others to do so, for Government purposes only.

(C) 2015 ACM 2329-4949/2015/01-ART11 $\$ 15.00$

DOI : http://dx.doi.org/10.1145/2686877 
reduce the communication costs of existing ones, even at the expense of doing more arithmetic.

Our motivation in this work is to reduce the communication costs when computing the eigenvalues and, optionally, eigenvectors, of a symmetric matrix. When starting from a full matrix, our sequential and distributed-memory parallel algorithms enable a communication-optimal symmetric eigensolver, whose overall communication costs asymptotically attain known lower bounds [Ballard et al. 2011b], up to logarithmic factors. When starting from a band matrix, it turns out that these lower bounds generally do not apply, and our new algorithms can communicate asymptotically less than both these lower bounds and previous algorithms. While we focus on tridiagonalization of symmetric matrices in this work, it is straightforward to extend our algorithms to the Hermitian case and to computing the singular value decomposition of a general (nonsymmetric, rectangular) matrix (e.g., via bidiagonalization).

Standard tridiagonalization-based approaches have two or three phases: (1) reduce the symmetric matrix to (symmetric) tridiagonal form by an orthogonal similarity transformation, (2) compute the eigenvalues and, optionally, eigenvectors of the tridiagonal matrix, and then (3), if eigenvectors are computed, transform their basis back by inverting the original orthogonal transformation. In this work, we focus on algorithms for phases (1) and (3).

For phase (1), we target a class of multistep tridiagonalization approaches collectively called successive band reduction (SBR) [Bischof et al. 2000a, 2000b] that generalize the classical one-step algorithm [Wilkinson 1962]: in each step, a symmetric band matrix is reduced to one with a narrower bandwidth by orthogonal similarity. Each step proceeds by an annihilate-and-chase approach, where annihilating entries within the band creates fill-in (bulges), and to preserve structure, bulges are chased off the band before annihilating subsequent entries. The motivation for a multistep approach is to improve efficiency: at least half of the arithmetic operations in the one-step approach are performed in communication-inefficient subroutines (e.g., BLAS-2 computations, like matrix-vector products) whose high communication costs often dominate the runtime. On the other hand, almost all of the arithmetic operations in a multistep approach can be performed with (communication-efficient) BLAS-3-like kernels. We will see in our analysis that, when computing only eigenvalues, a multistep approach is never detrimental to performance, and often quite beneficial, but when eigenvectors are additionally computed, the costs of phase (3) increase proportionally to the number of steps, leading to trade-offs between the costs of phase (1) and phase (3).

The main contribution of this work is extending the SBR design space to reduce communication costs. We refer to our new algorithms collectively as CommunicationAvoiding Successive Band Reduction, or CA-SBR. In particular, our contributions include the following.

- New Algorithms for Reducing Full Matrix to Band Form. In Section 3, we describe new sequential and distributed-memory parallel approaches for reducing a full symmetric matrix to band form (for the first step of phase (1)), combining the ideas of the Tall-Skinny QR algorithm [Demmel et al. 2012] and Householder reconstruction [Ballard et al. 2013a]. Their communication costs are optimal, asymptotically attaining lower bounds in [Ballard et al. 2011b] within logarithmic factors; moreover, the parallel algorithm asymptotically decreases the number of messages (on the critical path) compared to previous approaches.

- New Techniques for Avoiding Communication in Band Reduction. In Section 4, we present new techniques for avoiding communication in band reduction algorithms which require an annihilate-and-chase procedure. Our main contribution is the idea of chasing multiple bulges, which is novel in the context of band reduction. 
- New/Improved Sequential Algorithms. In Section 5, we give an asymptotic complexity analysis of previous approaches and show how our new techniques can be used to improve their communication costs. We also introduce a new algorithm that communicates asymptotically less than all other approaches.

- New Parallel Algorithm. In Section 6, we extend the ideas to a distributed-memory parallel algorithm that communicates asymptotically fewer messages than previous approaches.

A preliminary version of this work appeared in Ballard et al. [2012], considering the particular case of computing only the eigenvalues of a symmetric band matrix (with a constrained bandwidth). The multiple bulge chasing approach and sequential band reduction algorithm first appeared there; we also showed how to extend the sequential algorithm to a shared-memory parallel environment, demonstrating performance improvements over state-of-the-art library implementations. This article extends those results in three ways: we consider the reduction of full matrices to band form, discuss distributed-memory algorithms, and consider computing both eigenvalues and eigenvectors. However, we focus on theoretical analysis and do not give implementation details or performance results in this work.

The rest of the article is organized as follows. In Section 2 we establish necessary preliminaries, including notation, our communication model, and related work. Section 3 focuses on the step of reducing a full symmetric matrix to band form, which does not require the annihilate-and-chase procedure, in both sequential and parallel cases. Sections 4-6 focus on the reduction of a band matrix to tridiagonal form. The main techniques for avoiding communication in band reduction are described in Section 4, and we describe our application of those techniques to the sequential and parallel cases in Sections 5 and 6, respectively. We conclude in Section 7 with a discussion of our algorithms' limitations and possible extensions.

\section{PRELIMINARIES}

\subsection{Asymptotic Notation}

We use asymptotic notation $O(\cdot), \Theta(\cdot), \Omega(\cdot)$, and the slightly nonstandard $f \ll g, f \gg g$, to denote $f=o(g), f=\omega(g)$, respectively. All of our asymptotic expressions are to be interpreted as univariate, despite appearances. For example, $f=O\left(n^{2} b / M\right)$ asserts that, for every infinite sequence $\left(\left(n_{i}, b_{i}, M_{i}\right): i \in\{1,2, \ldots\}\right)$ of parameter triples satisfying certain given constraints, there exists constants $C \in(0, \infty)$ and $N \in\{1,2, \ldots\}$ such that $f \leq \mathrm{Cn}_{i}^{2} b_{i} / M_{i}$ for all $i \geq N$. The given constraints restrict the set of sequences of parameter triples with assumptions like $n b \gg M$ and $2 \leq b \leq \sqrt{M} / 3$, which are interpreted as univariate expressions $n_{i} b_{i} \gg M_{i}$ and $2 \leq b_{i} \leq \sqrt{M_{i}} / 3$. Phrases like asymptotically decrease are similarly constrained.

\subsection{Communication Model}

In order to quantify the communication costs of an algorithm, we model a sequential machine with two levels of memory hierarchy: a fast memory with an $M$ word capacity, and a slow memory of unbounded capacity. In our model, any number between 1 and $M$ of words can be read from or written to slow memory in a single message, provided the words are stored contiguously in slow memory. We count the number of words $W$ and messages $S$ moved between the two levels during the execution of the algorithm. This model is similar to the two-level I/O model [Hong and Kung 1981] and the disk access model [Aggarwal and Vitter 1988]. We also count the number $F$ of floating point operations (flops) performed; we ignore other arithmetic operations like index calculations, which have negligible cost in our algorithms. Supposing it takes the sequential 
machine $\gamma$ seconds per flop and $\alpha+\beta n$ seconds for an $n$-word message, then we can model the runtime $T$ as

$$
\max \{\gamma F, \beta W+\alpha S\} \leq T \leq \gamma F+\beta W+\alpha S,
$$

depending on the possibility of overlapping communication and computation. (The possibility of overlapping communication with itself is already modeled within the expression $\alpha+\beta n$.) We refer to $\gamma F, \beta W$, and $\alpha S$ as the arithmetic cost, bandwidth cost, ${ }^{1}$ and latency cost, respectively; the latter two are the communication costs.

In the distributed-memory parallel case, we model the machine as a collection of $p$ processors, each with a (limited) local memory of size $M$ and flop rate $1 / \gamma$, connected over a network with parameters $\alpha, \beta$. Processors communicate via point-to-point messages, again of size $n \in\{1, \ldots, M\}$ with cost $\alpha+\beta n$, and each processor can send or receive only one message at a time. The network topology is assumed to have allto-all connectivity, so we do not model contention or the number of hops a message would travel on a more realistic physical network. To model the parallel runtime $T$, we again use (1), except that we now measure $F, W$, and $S$ (the numbers of flops, words, and messages, respectively) along the critical path of the execution. For example, if two processors each send a message to separate processors simultaneously, the cost along the critical path (contribution to $T$ ) is that of one message (or less, if overlap is permitted).

In practice, in both sequential and parallel cases, $\gamma$ is much less than $\beta$ and $\beta$ is much less than $\alpha$, motivating our efforts to minimize communication.

In the rest of the article, in both the sequential and parallel case, we will be separately analyzing and considering $F, W$, and $S$ along the critical path as metrics for algorithmic performance. Our algorithms may benefit from overlapping of the various costs, potentially improving the runtime by a constant factor, and in practice they should be tuned to fully exploit this overlap. However, for the purposes of our asymptotic analysis, we will not discuss overlapping costs beyond this section.

We also note that, for a more refined model, the sequential and parallel cost models can be composed by measuring both sequential and parallel communication costs along the critical path in the parallel execution. Although we will not evaluate our algorithms in the composed model, we claim it is possible using our separate sequential and parallel analyses, because the arithmetic operations performed along the critical path in the parallel algorithm can be modeled as (portions of) the sequential algorithm. We leave open the study of overlapping and trading off sequential and parallel communication costs in a composed model; there is clearly a rich algorithmic design space.

\subsection{Symmetric Eigenproblems and Successive Band Reduction}

In this article, we are interested in computing the eigendecomposition $A=V \Lambda V^{T}$ of a symmetric matrix $A \in \mathbb{R}^{n \times n}$, that is, computing the eigenpairs $\left(v_{1}, \lambda_{1}\right), \ldots,\left(v_{n}, \lambda_{n}\right)$, where the eigenvector $v_{i}$ is the $i$ th column of the orthogonal matrix $V$ and its corresponding eigenvalue $\lambda_{i}$ is the $(i, i)$-entry of the diagonal matrix $\Lambda$. We will consider both the case where we compute $(V, \Lambda)$ and where we compute just $\Lambda$; additionally, our algorithms and analyses can be specialized to the case of computing a partial eigensystem, that is, a proper subset of the $n$ eigenpairs.

As explained in Section 1, we consider a class of algorithms for the symmetric eigenproblem that follow the two- or three-phase approach of (1) (multistep) tridiagonalization, (2) solving the tridiagonal eigenproblem, and (3) back-transformation, where

\footnotetext{
${ }^{1}$ To avoid confusion, we will always use the phrase "bandwidth cost" to differentiate this term from "bandwidth," which refers to the number of nonzero diagonals in a band matrix.
} 
phase (3) only applies to the case where both eigenvalues and eigenvectors are desired. We can express these computations as

$$
A=Q_{1} T Q_{1}^{T}=\left(Q_{1} Q_{2}\right) \Lambda\left(Q_{1} Q_{2}\right)^{T}=V \Lambda V^{T},
$$

where $Q_{1}$ defines the orthogonal similarity that reduces $A$ to the (symmetric) tridiagonal matrix $T$ in phase (1), $T=Q_{2} \Lambda Q_{2}^{T}$ is the eigendecomposition of $T$ computed in phase (2), and $V=Q_{1} Q_{2}$ is the eigenvector matrix of $A$ computed in phase (3).

As mentioned in Section 1, we focus on phases (1) and (3) in this work. In particular, we assume that phase (2) uses an efficient algorithm such as Bisection/Inverse Iteration [Barth et al. 1967], MRRR [Dhillon and Parlett 2004], Divide-and-Conquer [Cuppen 1980; Gu and Eisenstat 1992], or QR Iteration [Bowdler et al. 1968], and we ignore the computation and communication costs of this phase; for more discussion of tridiagonal eigensolvers, see Demmel et al. [2008].

Since the eigenvalues of $T$ are the eigenvalues of $A$, if only eigenvalues are desired, then $Q_{1}$ and $Q_{2}$ need not be computed or stored, that is, phase (3) (backtransformation) is unnecessary. However, if eigenvectors are also desired, then it is necessary to reconstruct them from the eigenvectors of $T$ by back-transformation, that is, evaluating the product $V=Q_{1} Q_{2}$. There are many possible ways to implement this computation. If we form $Q_{1}$ and $Q_{2}$ explicitly, then this can be done with matrix multiplication; if we store $Q_{1}$ implicitly, for instance, as a set of Householder vectors, then it can be applied to $Q_{2}$ after $Q_{2}$ is formed explicitly; if $Q R$ Iteration is used to compute the eigendecomposition of $T$, then $Q_{1}$ should be formed explicitly so that $Q_{2}$ can be applied implicitly to $Q_{1}$ from the right as it is computed; or $Q_{1}$ and $Q_{2}$ can be left implicit, allowing us to apply $V$ (to an explicit matrix) when needed. We will see that the back-transformation phase of our algorithms can dominate the overall runtime, and it is open whether the cost of back-transformation can be made independent of the number of (intermediate) band reductions performed in phase (1). ${ }^{2}$

As mentioned, we implement phase (1) with a multistep tridiagonalization approach, based on the successive band reduction (SBR) framework [Bischof et al. 2000b]; we will borrow notation from Bischof et al. [2000b] and the authors' related papers. Recall that an $n$-by- $n$ symmetric band matrix has bandwidth $b$ if its nonzeros are restricted to the $2 b+1$ principal diagonals (a full matrix has $b=n-1$ ). To exploit symmetry, we store and operate on only the lower triangle of the band matrix, though analogous algorithms apply to the upper triangle. When we refer to a column of the band, we mean the entries of the column on and below the diagonal. In a given step, SBR eliminates $d$ subdiagonals in sets of $c$ columns, ${ }^{3}$ using an annihilate-and-chase approach. We assume Householder transformations are used; each set of transformations eliminates a $d$-by-c parallelogram of nonzeros but creates trapezoidal-shaped fill-in (a bulge). Using analogous orthogonal similarities, SBR chases each bulge off the end of the band, translating the bulge $b$ columns to the right with each bulge chase. Figure 1 shows the data access pattern of a single bulge chase. A QR decomposition of the $(d+1+c)$-by-c matrix ( $Q R$ region in Figure 1) containing the parallelogram computes the orthogonal matrix that annihilates the parallelogram; the corresponding rows (PRE region) are updated with a premultiplication of the orthogonal matrix; the corresponding columns (POST region) are updated with a postmultiplication by the transpose of the orthogonal matrix, creating the next bulge; and the lower half of the corresponding symmetric submatrix on the diagonal (SYM region) is updated from both the left and right. (The

\footnotetext{
${ }^{2}$ However, we note that if only a subset of eigenpairs are desired, then the cost of the back-transformation can be made proportional to the number of eigenpairs desired.

${ }^{3}$ We depart from the LAPACK-style notation nb of Bischof et al. [2000b].
} 


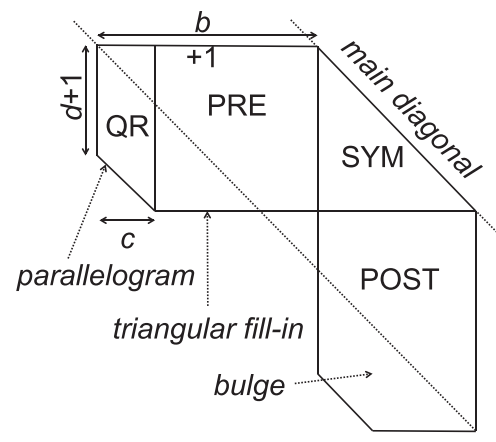

Fig. 1. Following the notation of Bischof et al. [2000b], the bulge chasing operation based on an orthogonal similarity transformation can be decomposed into four parts. There are $d$ diagonals in each bulge and $c$ is the number of columns annihilated during a bulge chase which leaves behind triangular fill-in.

reader may find Figure 2 in Bischof et al. [2000b] helpful to visualize how a step is comprised of a sequence of bulge chases, like the one depicted in Figure 1.)

We define the working bandwidth $b+d+1$ to be the number of subdiagonals necessary to store the $b+1$ diagonals of the matrix as well as to store the $d$ diagonals that hold temporary fill-in during the course of a step. As observed in Murata and Horikoshi [1975], we note that an entire bulge need not be eliminated; only the first $c$ columns of the bulge must be annihilated to prevent subsequent bulges from introducing nonzeros beyond the working bandwidth. This results in temporary triangular fill-in.

We index steps with an integer $i$, where $i=1$ is the first step, so $b_{1}=b$ is the initial bandwidth. We index the parallelograms which initiate each bulge chase by $j$ and the sequence of following bulges by the ordered pairs $(j, k): j$ is the parallelogram index and $k$ is the bulge index, as in Bischof et al. [1994].

\subsection{Related Work}

We survey previous sequential and parallel multistep algorithms for symmetric tridi- $^{4}$ agonalization and relevant extensions to bidiagonalization. We note that there are algorithms for the symmetric eigenproblem that do not reduce all the way to tridiagonal form and instead solve a band eigenproblem in phase (2) (see, e.g., [Gansterer et al. 1999; Haidar et al. 2012a]). There are also optimizations for the one-step tridiagonalization approach (see, e.g., [Blackford et al. 1997; Howell et al. 2008; Smith et al. 1994; Yamazaki et al. 2013]), but none asymptotically reduce communication. However, a survey of general approaches for the symmetric eigenproblem is beyond the scope of this article.

We note that many algorithms for the nonsymmetric eigenproblem begin by reducing the full matrix to Hessenberg form, and multistep approaches (see, e.g., Karlsson and Kågström [2011]) involve a similar type of bulge-chasing to that explored here, although the costs are substantially different.

Sequential Algorithms. The two papers of Bischof, Lang, and Sun [Bischof et al. $2000 \mathrm{a}, 2000 \mathrm{~b}$ ] provide a general framework of sequential SBR algorithms. Their approach first appeared in Bischof and Sun [1992] and generalizes most of the related work described in this section.

The annihilate-and-chase strategy began with Rutishauser and Schwarz in 1963. Rutishauser [1963] identified two extreme points in the SBR algorithm design space:

\footnotetext{
${ }^{4}$ All "symmetric" algorithms referenced or derived in this work can be readily generalized to the Hermitian case; we say "symmetric" throughout for brevity.
} 
(1) a Givens rotation-based approach with $b$ steps and $c_{i}=d_{i}=1$ for each $i$ and (2) a column-based approach with one step where $c_{1}=1$ and $d_{1}=b-1$. Rutishauser's first approach considered only pentadiagonal matrices; Schwarz [1963] generalized the algorithm to arbitrary bandwidths. Later, Schwarz [1968] proposed a different algorithm based on Givens rotations which does not fit in the SBR framework. This algorithm eliminates entries by column rather than by diagonal and does not generalize to parallelograms.

Murata and Horikoshi [1975] improved on Rutishauser's column-based algorithm by noting that computation can be saved by eliminating only the first column of the triangular bulge rather than the entire triangle. If eigenvectors are desired, Bischof et al. [1994] showed that, with this approach, the Householder vectors comprising $Q_{1}$ can be stored in a lower triangular $n$-by- $n$ matrix and applied to $Q_{2}$ in a different order than they were computed, yielding higher performance during the backtransformation phase.

Kaufman [1984] vectorized the Rutishauser/Schwarz algorithm [Rutishauser 1963; Schwarz 1963], chasing multiple single-element bulges in each vector operation. Her motivation for chasing multiple bulges was not locality but rather to increase the length of the vector operation beyond the bandwidth $b$. Several years later, Kaufman [2000] took the approach of Schwarz [1968] in order to maximize the vector operation length (especially in the case of large $b$ ) and make use of a BLAS-1-like subroutine when appropriate. When eigenvectors are requested, the matrix $Q$ is formed explicitly by applying the updates to an identity matrix. By exploiting sparsity, the flop cost of constructing $Q$ is about $(4 / 3) n^{3}$, compared with $2 n^{3}$ if sparsity is ignored. The current LAPACK [Anderson et al. 1992] reference code for band reduction (sbtrd) is based on Kaufman [2000].

More recently, Rajamanickam [2009] proposed and implemented a different way of eliminating a parallelogram and chasing its fill-in. His algorithm uses Givens rotations to eliminate the individual entries of a parallelogram, and instead of creating a large bulge, the update rotations are pipelined such that as soon as an element is filled in outside the band, it is immediately annihilated. The rotations are carefully ordered to obtain temporal and sequential locality. By avoiding the fill-in, this algorithm does up to $50 \%$ fewer flops than the Householder-based elimination of parallelograms within SBR and requires minimal working bandwidth ( $b+1$ subdiagonals).

Parallel Algorithms. Lang [1991, 1993] implemented a distributed-memory parallel version of the band reduction algorithm in Murata and Horikoshi [1975], although he did not consider computing $Q$. He later extended the approach to bidiagonalization $A=U B W^{T}$ of band matrices [Lang 1996], and gave four different approaches for computing the orthogonal matrices $U$ and $W$ (which also apply to computing $Q$ in the symmetric case). This was extended to two-step bidiagonalization of full matrices in distributed-memory [Großer and Lang 1999] and shared-memory [Lang 1999]. Bischof et al. [1993] implemented a distributed-memory parallel instance of the SBR framework in the context of tridiagonalizing a full matrix. They used a two-step approach which blocked the Householder updates in the first (full-to-band) step, and then directly tridiagonalized the remaining band. A subsequent paper [Bischof et al. 1994] extended this implementation to reorganize and block the orthogonal updates from the second step.

Luszczek et al. [2011] implemented a two-step shared-memory tridiagonalization algorithm in the PLASMA library [Agullo et al. 2009], using dynamic DAG-scheduling of tile-based tasks. They used tiled kernels to obtain high performance in the first step, and parallelized the band reduction algorithm from Murata and Horikoshi [1975] for the second step. They distinguished between "right-looking" and "left-looking" 
variants: right-looking algorithms chase a bulge entirely off the band before eliminating the next parallelogram, left-looking algorithms chase bulges only far enough to allow for the next bulge to be created (see Constraint 4.2 in Section 4.2). For example, the SBR framework [Bischof et al. 2000b] is right-looking while Kaufman's algorithm [Kaufman 2000] is left-looking. Luszczek et al. [2011] found improved performance with a left-looking variant. Later, Haidar et al. [2011] improved the runtime of Luszczek et al. [2011] by enabling the first and second steps to exploit different tile sizes, and by fusing certain kernels in both steps; moreover, in the second step, they employed an algorithm-specific (static) scheduler and avoided fill-in using pipelined Givens rotations (a single-step version of the approach in Rajamanickam [2009]). Efficient performance on the first (full-to-band) step has been obtained by offloading it to one GPU [Bientinesi et al. 2010] or many [Haidar et al. 2013b]. (This work has also been extended to bidiagonalization-based approaches for the SVD [Haidar et al. 2012b, 2013a; Ltaief et al. 2013].)

Auckenthaler [2012] and Auckenthaler et al. [2011a, 2011b] have implemented a two-step distributed-memory tridiagonalization algorithm as part of a solver for the generalized symmetric eigenproblem. Their band-to-tridiagonal step used an improved version of Lang's algorithm [Lang 1993] (which performs one step). They gave a new algorithm for orthogonal updates which used a 2D processor layout instead of a 1D layout (first suggested in Lang [1996], for the case of bidiagonalization). Their implementation also supported taking more than 2 steps when eigenvectors are not requested; however, this algorithm was not given explicitly.

\subsection{Related Lower Bounds}

No communication lower bound has been established for annihilate-and-chase band reduction algorithms, so we cannot conclude that our new algorithms are communication-optimal in an asymptotic sense. In fact, the communication lower bounds in Ballard et al. [2011b], which apply widely in numerical linear algebra, including classical algorithms for matrix multiplication and many orthogonal factorizations, do not apply to general band reduction algorithms because they fail to satisfy "forward progress" [Ballard et al. 2011b, Definition 4.3]. That is, the lower bound assumes that an orthogonal transformation algorithm does not fill in entries after they have been eliminated, but this occurs frequently in SBR, unlike one-sided orthogonal factorizations like the $\mathrm{QR}$ decomposition.

The main result of Ballard et al. [2011b] states that an applicable algorithm that performs $G$ flops must move $\Omega(G / \sqrt{M})$ words and send $\Omega\left(G / M^{3 / 2}\right)$ messages for sufficiently large problems. For most dense matrix algorithms, the number of flops is $G=O\left(n^{3} / p\right)$, where $p=1$ for the sequential case. In the parallel case, if we assume minimal local memory is used (i.e., $M=\Theta\left(n^{2} / p\right)$, or just enough to store the input and output matrices), the lower bounds simplify to $\Omega\left(n^{2} / \sqrt{p}\right)$ words and $\Omega(\sqrt{p})$ messages.

Since our new sequential algorithm (see Algorithm 1 and Table III) performs $O\left(n^{2} b\right)$ flops, moves $O\left(n^{2} b^{2} / M\right)$ words, and sends $O\left(n^{2} b^{2} / M^{2}\right)$ messages, its bandwidth and message counts drop below the lower bounds by a factor of $O(\sqrt{M} / b)$ for $2 \leq b \leq \sqrt{M} / 3$. For small $b$ and large $n$ (such that the band does not fit entirely in fast memory), the factor by which our algorithms beat this (inapplicable) lower bound is as much as $O(\sqrt{M})$. Similarly, our new parallel algorithm (see Algorithm 3 and Table V) also beats the lower bounds for bandwidth and message counts, and the factor is largest for small bandwidths. Thus, our algorithms show that not only does the lower bound proof technique not apply to annihilate-and-chase algorithms, the bound itself must not apply, that is, an assumption like forward progress is necessary. 
It is important to note that the lower bounds do apply to any step of a band reduction algorithm with $b>n / 2$ and $d$ chosen appropriately, for instance, any full-to-band step, as the computations in that step satisfy forward progress. In fact, the approaches we propose in Section 3 attain the bounds and are therefore communication optimal (at least to within logarithmic factors). By showing that the costs of subsequent steps do not dominate this first step, we can claim that the overall tridiagonalization algorithm (starting with a full matrix) is communication optimal.

\section{AVOIDING COMMUNICATION IN REDUCING SYMMETRIC MATRICES TO BAND FORM}

Recall that the first step in a multistep approach applied to a full symmetric matrix is to reduce to band form. As noted in Bischof et al. [2000b], almost all of the work in this first (full-to-band) step can be cast in terms of matrix multiplication, maximizing data reuse in the sequential case. In this section, we discuss previous approaches for reducing full symmetric matrices to band form in both the sequential and parallel cases, and we also discuss our new approach. Throughout, we assume that $n^{2} \gg M$, that is, the problem is sufficiently large with respect to the fast/local memory size $M$.

In the case that eigenvectors $V$ are desired, the orthogonal transformation $Q$ that reduces the full matrix to band form will be applied to the eigenvector matrix $W$ of the band matrix during the back-transformation phase, that is, $V=Q W$. Applying from its implicit representation as a lower-triangular matrix of Householder vectors, as computed by the algorithms in this section, requires $2 n^{3}$ flops, $O\left(n^{3} / \sqrt{M}\right)$ words, and $O\left(n^{3} / M^{3 / 2}\right)$ messages in serial [Ballard et al. 2011a], and $2 n^{3} / p$ flops, $O\left(n^{2} / \sqrt{p}\right)$ words, and $O\left(\sqrt{p} \log ^{2} p\right)$ messages in parallel [Auckenthaler 2012], using the standard blocked algorithms. These costs can be diminished in the case of computing a partial eigensystem, where $W$ has fewer than $n$ columns. However, $Q$ in its implicit form requires $\Theta\left(n^{2}\right)$ storage, and in some scenarios, it may be desirable to form $Q$ explicitly and then apply the orthogonal transformations from subsequent band-reduction steps or eigensolves immediately to $Q$.

\subsection{Panel Factorizations and Symmetric Trailing Matrix Update}

The main bottleneck in direct (one-step) tridiagonalization of a full matrix is that, for each column in the matrix, a BLAS-2 operation involving the entire trailing matrix needs to be performed before computing the Householder vector that eliminates the column. The advantage of reducing a symmetric matrix to band form (bandwidth $b>$ 1) rather than tridiagonal form is that that the first $b$ columns can be eliminated (below the band) without accessing the trailing matrix. That is, we can perform an isolated QR decomposition on the subpanel of width $b$ (below the $b \times b$ diagonal block), and then update the symmetric trailing matrix with matrix multiplications, essentially a blocked version of the direct tridiagonalization approach [Bischof et al. 1994]. While blocking techniques applied to direct tridiagonalization can cast about half the work in terms of BLAS-3 computations [Dongarra et al. 1989], reducing to band form allows almost all of the work to be performed with BLAS-3 subroutines. The corresponding routine for this operation is syrdb in the sequential SBR framework [Bischof et al. 2000a].

However, note that the panel QR factorizations, while constituting only $O\left(n^{2} b\right)$ flops (a lower order term), can introduce a communication bottleneck. In particular, using Householder QR for the panel factorizations results in suboptimal number of messages in both sequential and parallel cases. One solution to this problem is to use a more communication-efficient algorithm for the panel factorization: Tall-Skinny QR (TSQR) [Demmel et al. 2012]. TSQR minimizes both word and message counts, alleviating the communication bottleneck, but the implicit representation of the orthogonal factor has 
a different structure than Householder QR, requiring a different method for the twosided update. A shared-memory parallel implementation of this approach is given in [Luszczek et al. 2011], which we discuss in more detail in Section 3.2.

We propose an alternate scheme for the full-to-band step, using a technique known as Householder reconstruction [Ballard et al. 2013a]. It is possible to perform TSQR and then convert the implicit storage of the orthogonal factor to a set of Householder vectors for only about 2.5 times the cost of the TSQR itself (requiring asymptotically the same number of flops, words, and messages). ${ }^{5}$ Because the arithmetic in all the panel factorizations amounts to only $O\left(n^{2} b\right)$ flops, the increase does not affect the leading constant in the overall flop count. This method enables the panel factorization to be performed efficiently with respect to communication but also allows for reuse of the software infrastructure to update the trailing matrix with matrix multiplications involving standard Householder representations. Because there are known lower bounds for this computation [Ballard et al. 2011b], we argue in Sections 3.2 and 3.3 that our approach is communication optimal.

\subsection{Sequential Case}

For the sequential case, we consider two alternative approaches: the original SBR framework [Bischof et al. 2000a] and the PLASMA library [Luszczek et al. 2011]. In order to minimize the number of words moved in an asymptotic sense, the reduced bandwidth of the matrix must be chosen to be $b=\Theta(\sqrt{M})$ for all the algorithms. For comparison, we note that the cost of direct tridiagonalization includes $O\left(n^{3}\right)$ words moved and at least $O\left(n^{3} / M\right)$ messages.

The SBR framework uses column-major ordering, and it uses the standard Householder QR algorithm within LAPACK [Anderson et al. 1992] for the panel factorization. The precise word and message counts of the panel factorizations depend on the relative sizes of $n$ and $M$; we assume for simplicity here that communication in the panel factorizations does not contribute to the leading order terms. The trailing matrix updates, which involve matrix multiplications, require $(4 / 3) n^{3}$ flops. Furthermore, because $b$ is chosen to be proportional to $\sqrt{M}$, the trailing matrix updates move $O\left(n^{3} / \sqrt{M}\right)$ words, which is optimal. However, because the matrix is stored in column-major order, the message count in the matrix multiplications amounts to $O\left(n^{3} / M\right)$, exceeding the lower bound by a factor of $\Theta(\sqrt{M}) .^{6}$ These counts appear in the first row of Table I.

The PLASMA library uses a block-contiguous storage format for the matrix and TSQR for the panel factorization. (While the algorithm described in Luszczek et al. [2011] is designed for multicore machines, we consider its serialization as a sequential, tiled algorithm.) In a block-contiguous format, the matrix is stored as a matrix of $b$-by$b$ contiguous submatrices; here the size of the blocks $b$ coincides with the bandwidth of the reduced matrix. The advantage of this format is that block operations involve $O\left(b^{3}\right)$ flops, $O\left(b^{2}\right)$ words, and $O(1)$ messages; with $b=\Theta(\sqrt{M})$, this corresponds to optimal communication costs overall. Since all of the operations (both panel factorizations and trailing matrix updates) are block operations, and there are a total of $O\left(n^{3} / b^{3}\right)$ block operations, the total flop, word, and message counts are easily computable and are given in the second row of Table I.

\footnotetext{
${ }^{5}$ Note also that there is no cost in terms of loss of numerical stability; the method is as stable as Householder QR and TSQR.

${ }^{6}$ Note that the SBR framework can be implemented for non-column-major storage formats; however, the use of Householder QR introduces a trade-off between the message count of the panel factorization and the message count of the trailing matrix update. See [Ballard et al. 2013b] for more discussion of this trade-off.
} 
Table I.

We compare previous sequential algorithms for reducing a full symmetric matrix to band form (bandwidth $b=\Theta(\sqrt{M})$ ) with our improvements. In the last row we give the communication lower bounds from [Ballard et al. 2011b].

\begin{tabular}{|c|c|c|c|}
\hline Algorithm & Flops & Words & Messages \\
\hline SBR [Bischof et al. 2000b] & $\frac{4}{3} n^{3}$ & $O\left(\frac{n^{3}}{\sqrt{M}}\right)$ & $O\left(\frac{n^{3}}{M}\right)$ \\
\hline PLASMA [Luszczek et al. 2011] & $\frac{4}{3} n^{3}$ & $O\left(\frac{n^{3}}{\sqrt{M}}\right)$ & $O\left(\frac{n^{3}}{M^{3 / 2}}\right)$ \\
\hline CA-SBR & $\frac{4}{3} n^{3}$ & $O\left(\frac{n^{3}}{\sqrt{M}}\right)$ & $O\left(\frac{n^{3}}{M^{3 / 2}}\right)$ \\
\hline Lower Bound & - & $\Omega\left(\frac{n^{3}}{\sqrt{M}}\right)$ & $\Omega\left(\frac{n^{3}}{M^{3 / 2}}\right)$ \\
\hline
\end{tabular}

Our proposed approach, which we refer to as CA-SBR, also uses a block-contiguous storage format, but instead uses TSQR and Householder reconstruction for the panel factorizations. The flop, word, and message counts for the panel factorization are derived in Ballard et al. [2013a], for the parallel case. In the sequential case, the panel factorization consists of at most three block operations for each block in the panel (compared to one in the case of TSQR). The trailing matrix update, which takes the same form of matrix multiplications as in the SBR framework, is also done with block operations. Since a block-contiguous format is used, not only is the word count minimized, the message count is minimized as well. The counts for our approach are given in the third row of Table I.

Note that both PLASMA and CA-SBR are communication optimal for the full-toband step. The disadvantage of CA-SBR is that the number of flops, words, and messages in the panel factorization are constant factors larger that those of PLASMA. However, the extra flops amount to only a lower order term in the overall cost of the step, and the extra words and messages do not affect the overall asymptotic counts. Another disadvantage of CA-SBR in the shared-memory parallel environment is that the panel factorization must be completed before the trailing matrix update begins; PLASMA allows significant overlap between these parts of the computation. The main advantage of CA-SBR is that recovering the implicit Householder form allows for better performance portability and possibly better performance. By casting the trailing matrix update in terms of matrix multiplication rather than a set of block kernels (each of which must be tuned for performance), we can expect practical benefits from the thorough tuning of matrix multiplication kernels on every architecture.

We also consider in this section the cost of converting the full matrix storage format to the band format we require in subsequent steps. Because the optimal algorithms full-to-band algorithms (PLASMA and CA-SBR) require block-contiguous storage, we must convert the remaining band to LAPACK symmetric band storage (see Section 5.1.2). At the end of the first step, each set of $b$ columns is stored in two blocks: one symmetric diagonal block and one triangular off-diagonal block. Thus, the transformation requires one pass through the band: communicating $O(n b)$ words and, since blocks are accessed contiguously, $O(n / b)$ messages, both lower order terms.

\subsection{Parallel Case}

In the distributed-memory parallel case, we compare our approach with the original parallel SBR algorithm [Bischof et al. 1994], which is used in the ELPA library [Auckenthaler 2012; Auckenthaler et al. 2011a, 2011b]. Also, we note that direct tridiagonalization moves $O\left(n^{2} / \sqrt{p}\right)$ words and $O(n \log p)$ messages, and it suffers from poor on-node performance due to the use of local BLAS-2 subroutines. 
Table II.

We compare previous parallel algorithms for reducing a full symmetric matrix to band form (bandwidth $b=O(n / \sqrt{p})$ ) with our improvements. In the last row we give the communication lower bounds from [Ballard et al. 2011b], assuming $M=O\left(n^{2} / p\right)$.

\begin{tabular}{|c|c|c|c|}
\hline Algorithm & Flops & Words & Messages \\
\hline SBR [Bischof et al. 1994; Auckenthaler 2012] & $\frac{4}{3} \frac{n^{3}}{p}$ & $O\left(\frac{n^{2}}{\sqrt{p}}\right)$ & $O(n \log p)$ \\
\hline CA-SBR & $\frac{4}{3} \frac{n^{3}}{p}$ & $O\left(\frac{n^{2}}{\sqrt{p}}\right)$ & $O\left(\sqrt{p} \log ^{2} p\right)$ \\
\hline Lower Bound & - & $\Omega\left(\frac{n^{2}}{\sqrt{p}}\right)$ & $\Omega(\sqrt{p})$ \\
\hline
\end{tabular}

The algorithm described in Bischof et al. [1994] for reducing the full matrix to band form uses a block-cyclic data distribution, with block size equal to the bandwidth of the reduced matrix. As in the sequential case, it uses the standard Householder QR algorithm (as implemented in ScaLAPACK [Blackford et al. 1997]). More precise cost analysis of the parallel SBR algorithm is given in Auckenthaler [2012], which we present in the first row of Table II. Note that we omit a logarithmic factor in the word count, assuming optimal collectives are used within the algorithm (tree-based collectives are assumed in Auckenthaler [2012]). Assuming the local memory used is $M=O\left(n^{2} / p\right)$ (i.e., no more than a constant factor more than what is required to store the input matrix), this algorithm is optimal with respect to the word count, matching the known lower bound [Ballard et al. 2011b]. However, the message count, $O(n \log p)$, exceeds the lower bound of $\Omega(\sqrt{p})$ by a significant factor. The $O(n \log p)$ term is a result of the parallel reductions required to compute a Householder vector for each subcolumn of the matrix (within the panel factorizations). ${ }^{7}$

Our CA-SBR approach, based on Householder reconstruction, removes the latency overhead of Householder QR and maintains efficient trailing matrix updates similar to the SBR approach. We also assume a block-cyclic data layout with block size $b=n /(p \log p)$ equal to the bandwidth of the reduced matrix. The analysis of the parallel panel factorization using Householder reconstruction is given in Ballard et al. [2013a], and the counts for the update are the same as in Auckenthaler [2012]. The total counts of the CA-SBR approach appear in the second row of Table II; assuming $M=O\left(n^{2} / p\right)$, the algorithm is communication optimal (to within logarithmic factors in terms of latency). The performance benefit of blocking the trailing matrix update for the case of (one-sided) QR factorization is demonstrated in the distributed-memory parallel case in Ballard et al. [2013a].

Note that TSQR can be used without Householder reconstruction, but the trailing matrix update would need to be performed in a manner similar to the shared-memory implementation of PLASMA discussed in Section 3.2. We have not analyzed this algorithm, but we expect the counts to be within logarithmic factors of the lower bounds.

Finally, we analyze redistributing the band matrix from the $2 \mathrm{D}$ block-cyclic layout to a $1 \mathrm{D}$ block layout required by the band reduction algorithm (see Section 6.1.2). As in the sequential case, after reducing to band form, each set of $b$ columns is split into a symmetric diagonal block and a triangular off-diagonal block (owned by two processors in the same processor column). In order to achieve a 1D block-cyclic distribution across $\sqrt{p}$ processors with the same block size, pairs of processors in the same column can exchange data for negligible cost. Note that we assume only $\sqrt{p}$ processors are used for the band reduction phase if eigenvectors are required. The CA-SBR band reduction

\footnotetext{
${ }^{7}$ Auckenthaler also proposes a variant of Cholesky-QR to alleviate this latency bottleneck [Auckenthaler 2012], but because it weakens the numerical stability of the algorithm, we ignore this performance optimization here.
} 
algorithm can be adapted to a block-cyclic layout, but we will assume a redistribution to a block layout is required. In order to redistribute the block-cyclic layout to a block layout (or any other layout for that matter), we perform an all-to-all collective among the processors owning the band. The counts for this all-to-all, assuming we use the variant that minimizes latency, are $O(n b \log p / \sqrt{p})=O\left(n^{2} / \sqrt{p}\right)$ words and $O(\log p)$ messages [Bruck et al. 1994], neither of which affects the overall asymptotic counts.

\section{AVOIDING COMMUNICATION IN BAND REDUCTION}

In this section and the following two, we will focus on reducing a band matrix to tridiagonal form. The goal of our band reduction algorithms is to avoid communication by reorganizing computation, extending the SBR framework to obtain greater data locality. In the sequential case, we can asymptotically reduce the number of words and messages that must be moved between fast and slow memory during the execution of the algorithm; in the parallel case, we can asymptotically reduce the number of messages sent between processors. We achieve data locality (i.e., avoid communication) by applying multiple Householder transformations and by chasing multiple bulges, as described in Sections 4.1 and 4.2. We navigate the constraints and trade-offs that arise using a successive halving approach, described in Section 4.3.

It is important to note that when both eigenvalues and eigenvectors are computed, the costs of phase (3) (back-transformation) increase proportionally to the number of steps in the (multistep) tridiagonalization approach in phase (1). In particular, our proposed (multistep) successive halving approach (Section 4.3) increases backtransformation costs by factors of $O(\log (b)$ ) (see Tables IV and VI), and for some ranges of parameters, the additional costs can dominate the benefits of reducing communication in phase (1). This trade-off has always been used as a counterargument against multistep approaches in favor of one-step approaches, yet the prevalence of high-performance two-step implementations, starting with [Bischof et al. 1993], have made the case that this trade-off is worth exploring.

\subsection{Applying Multiple Householder Transformations}

The first means of achieving data locality is within a single bulge chase (see Figure 1). This technique is enabled by the flexibility of the original SBR framework. By eliminating parallelograms of width $c$ rather than single columns, we are able to perform multiple computations on the same data. Since $c$ Householder vectors are computed to eliminate the first $c$ columns of the bulge ( $Q R$ region), every entry in the PRE, SYM, and POST regions is updated by $c$ left and/or right Householder transformations. These transformations may be applied one at a time or blocked (e.g., via compact-WY representations [Schreiber and Van Loan 1989]). Assuming all the data involved in a single bulge chase reside in fast or local memory, $O(c)$ flops are performed for every entry read from slow memory.

We identify the following algorithmic constraint. If it is violated, then the parallelogram annihilated by the left update will be (partially) refilled-in by the right update (i.e., the SYM and POST regions overlap the QR region)—this implies wasted computation.

Constraint 4.1. To annihilate a parallelogram within the SBR framework, the dimensions of the parallelogram must satisfy

$$
c+d \leq b .
$$

While increasing $c$ improves data locality, it limits the size of $d$ due to Constraint 4.1. Because $d$ is the number of diagonals eliminated in a step, this constraint creates a trade-off between locality and progress towards tridiagonal form. 


\subsection{Chasing Multiple Bulges}

The second means of achieving data locality is across bulge chases. If $\omega$ bulges can be chased through the same set of columns without data movement, then we have achieved $O(\omega)$ reuse of those columns. This technique of chasing multiple bulges (without overlapping) has been used in the context of Hessenberg QR iteration for the nonsymmetric eigenproblem; see Braman et al. [2002] and Granat et al. [2010].

Recall that we refer to columns as the subset of column entries on and below the diagonal. We first establish the following constraint.

Constraint 4.2. No bulge may be chased into a set of columns still occupied by a previously created bulge.

If this constraint is violated, then the fill-in will expand beyond the working bandwidth of the step. While it is possible to eliminate this extra fill-in, we wish to avoid the extra arithmetic, communication, and storage it seems to require. Chasing the first $c$ columns of a bulge and leaving behind the triangular fill-in is the least amount of work required to prevent the fill-in from exceeding the working bandwidth.

We state the following lemmas regarding parallelograms, bulges, sets of bulges, and the working set (measured in columns) for chasing a set of bulges. We assume in both cases that Constraints 4.1 and 4.2 are satisfied.

LEMMA 4.3. Given a step of SBR with parameters $b$, $c$, and $d$,

(a) the jth parallelogram occupies columns $1+(j-1) c$ through jc,

(b) bulge $(j, k)$ occupies columns $1+(j-1) c+k b-d$ through $j c+k b$,

(c) bulges $(j, k)$ and $(j+1, k-2)$ do not overlap. ${ }^{8}$

LEMMA 4.4. Chasing the set of $\omega$ bulges

$$
\{(j, k),(j+1, k-2), \ldots,(j+\omega-1, k-2(\omega-1))\}
$$

each $\ell$ times requires a working set of $(\omega-1)(2 b-c)+c+d+b \ell$ columns.

PROOF. By Lemma 4.3(c), this set of bulges is nonoverlapping. If the bulges are chased in turn $\ell$ times each, starting with the right-most bulge $(j, k)$ and ending with the left-most bulge $(j+\omega-1, k-2(\omega-1))$, then there is no violation of Constraint 4.2. The conclusion follows from Lemma 4.3(b).

Figure 2 demonstrates the working set of 44 columns with $\omega=2$ bulges chased $\ell=3$ times each on a matrix with bandwidth $b=8$ with $c=d=4$.

Our motivation for defining a working set is to ensure that the operation of chasing $\omega$ bulges $\ell$ times can be done entirely in fast memory (in the sequential case) or local memory (in the parallel case). We will specify the constraints in each case when we present our algorithms below.

\subsection{Successive Halving}

We will navigate the trade-off imposed by Constraint 4.1 by setting $c_{i}=d_{i}=b_{i} / 2$ at each step $i$, reducing to tridiagonal form after $\log b$ steps. We call this a successive halving approach. We will pick the number of bulges in a set $\left(\omega_{i}\right)$ and the number of times each bulge is chased $\left(\ell_{i}\right)$ such that on each step (as the bandwidth is successively halved) we double the number of bulges that we chase in a set, and chase each bulge twice as many times, compared to the previous step.

While the successive halving approach (and doubling $\omega_{i}$ and $\ell_{i}$ ) simplifies our asymptotic analysis, in practice the parameters $\left\{c_{i}, d_{i}, \omega_{i}, \ell_{i}\right\}$ should be tuned independently

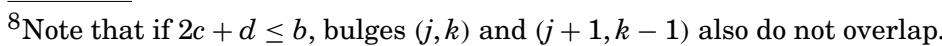




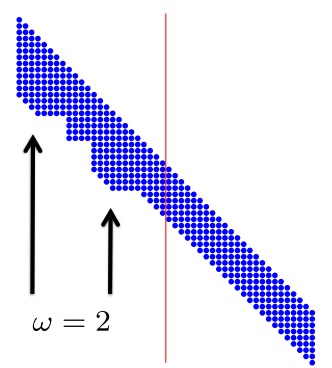

(a) The $\omega=2$ bulges occupy 20 of the 24 columns on the left.

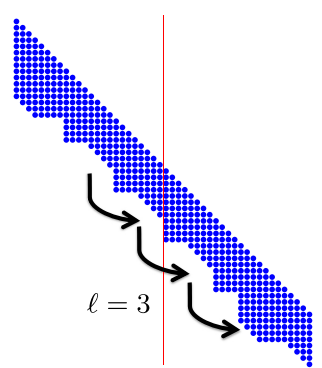

(b) The right-most bulge is chased $\ell=3$ times.

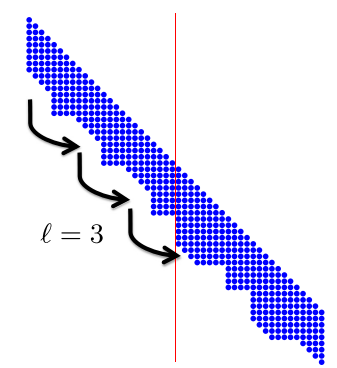

(c) The second bulge is chased $\ell=3$ times.

Fig. 2. This figure demonstrates chasing a set of bulges. We store and operate on only the lower triangle of the band matrix. The parameters shown are $b=8, c=4$, and $d=4 ; \omega=2$ bulges are chased $\ell=3$ times each. Only $2 b \ell=48$ columns of the band are shown. The working bandwidth includes the diagonals which contain bulges and triangular fill-in. Note that the triangular fill-in left behind by the first bulge does not cause any increase in the working bandwidth as the second bulge is chased.

for best performance; we previously suggested a framework for automatically tuning these parameters in a shared-memory implementation [Ballard et al. 2012, Section 5], although it did not consider the case of computing both eigenvalues and eigenvectors, as described earlier, where the trade-offs from taking multiple steps are more severe. Ultimately, this asymptotic analysis can be extended to develop more refined performance models; by fixing certain architectural parameters, one can then evaluate whether certain terms, like factors of $\log b$, are significant.

\section{SEQUENTIAL BAND TRIDIAGONALIZATION ALGORITHMS}

Recall our sequential machine model, where communication is moving data between slow memory of unbounded capacity and a fast memory with a capacity of $M$ words. We will first consider the case of computing eigenvalues only and then extend to the case of computing both eigenvalues and eigenvectors. We will not analyze the solution of the tridiagonal eigenproblem. In each case, we discuss existing approaches, apply our techniques to improve them, and then present our communication-avoiding approach.

For our sequential algorithms, we will assume the initial bandwidth $b$ is bounded above by $\sqrt{M} / 3$. This is a reasonable assumption after the first step in a multistep tridiagonalization approach, starting from a full matrix (see Section 3). We do not treat the case when the input matrix has an intermediate bandwidth $\sqrt{M} / 3<b<n-1$, although we claim that it is asymptotically optimal to take a single sweep of band reduction, reducing to bandwidth at most $\sqrt{M} / 3$ while exploiting blocked kernels, and then applying the band algorithms in this section. We also assume that $n b \gg M$ (the band does not fit in fast memory).

\subsection{Computing Eigenvalues Only}

When only eigenvalues are desired, the runtime is dominated by the band reduction. Computing the eigenvalues of a tridiagonal matrix involves only $O(n)$ storage and less computation than the band reduction; $O\left(n^{2}\right)$ flops as opposed to $O\left(n^{2} b\right)$. While there is a large design space for band reduction, the (leading-order) flop count ranges from $4 n^{2} b$ to $6 n^{2} b$, a difference of only $50 \%$ (as long as a bulge-chasing procedure is used to prevent unnecessary fill-in). However, the word and message counts (and expected performance) have much larger ranges.

Under the assumption $n b \gg M$, the matrix does not fit in fast memory (otherwise, the word counts are the same for all algorithms: $O(n b))$. In the case that $n<M$ (i.e., 
Table III.

We compare previous sequential algorithms for tridiagonalization (for eigenvalues only) with our improvements, for symmetric band matrices of $n$ columns and $b+1$ subdiagonals on a machine with fast memory of size $M$. The table assumes that $n b \gg M$ and that $2 \leq b \leq \sqrt{M} / 3$. The analysis for all algorithms is given in Section 5. In the fourth and fifth rows, $s$ is the number of steps performed and $t \leq s$ is the smallest step index such that the subsequent steps can be performed in fast memory, or $t=s$ otherwise.

\begin{tabular}{|c|c|c|c|}
\hline Algorithm & Flops & Words & Messages \\
\hline LAPACK [Kaufman 2000] & $4 n^{2} b$ & $O\left(n^{2} b\right)$ & $O\left(n^{2} b\right)$ \\
\hline MH [Murata and Horikoshi 1975] & $6 n^{2} b$ & $O\left(n^{2} b\right)$ & $O\left(\frac{n^{2} b}{M}\right)$ \\
\hline Improved MH & $6 n^{2} b$ & $O\left(\frac{n^{2} b^{3}}{M}\right)$ & $O\left(\frac{n^{2} b^{3}}{M^{2}}\right)$ \\
\hline SBR [Bischof et al. 2000b] & $\sum_{i=1}^{s}\left(4 d_{i}+2 \frac{d_{i}^{2}}{b_{i}}\right) n^{2}$ & $O\left(\sum_{i=1}^{t}\left(1+\frac{d_{i}}{b_{i}}\right) n^{2}\right)$ & $O\left(\sum_{i=1}^{t}\left(1+\frac{d_{i}}{b_{i}}\right) \frac{n^{2}}{M}\right)$ \\
\hline SBR $\left(c_{i}=d_{i}=b_{i} / 2\right)$ & $5 n^{2} b$ & $O\left(n^{2} t\right)$ & $O\left(\frac{n^{2} t}{M}\right)$ \\
\hline CA-SBR & $5 n^{2} b$ & $O\left(\frac{n^{2} b^{2}}{M}\right)$ & $O\left(\frac{n^{2} b^{2}}{M^{2}}\right)$ \\
\hline
\end{tabular}

one or more diagonals fit in fast memory), when the bandwidth is reduced such that the remaining band matrix fits in fast memory, the communication cost of remaining steps is that of reading the band into fast memory once and writing the tridiagonal output.

Table III summarizes the flop, word, and message counts of various algorithms for tridiagonalizing a band matrix (for computing eigenvalues only), which are presented and analyzed in Sections 5.1.1 and 5.1.2. Our new approach, CA-SBR (Algorithm 1), improves the communication costs compared to the previous approaches. For example, CA-SBR moves a factor of $M / b$ fewer words than LAPACK or $\mathrm{MH}$, which is at least $\sqrt{M}$ in the range of $b$ considered, and near $M$ for $b=O(1)$. If we start with a full matrix, using CA-SBR for the band-to-tridiagonal step(s) always attains (or beats) the lower bounds discussed in Section 2.5. See Section 5.2 for more discussion.

5.1.1. Alternative Algorithms. We first consider Kaufman's algorithm [Kaufman 2000], which is implemented in the current LAPACK reference code [Anderson et al. 1992], given in the first row of Table III. The algorithm uses Givens rotations and performs $4 n^{2} b$ flops. It is left-looking and chases multiple single-element bulges in order to maximize the vector operation length, but it does not limit the size of the working set to fit in fast memory. As a result, the algorithm has to read (from slow memory) at least one of each pair of entries to be updated by a Givens rotation. Thus, the data reuse is $O(1)$ and the total number of words transferred between fast and slow memory is proportional to the number of flops: $O\left(n^{2} b\right)$. Since fine-grained data access occurs along both rows and columns, the message count is on the same order as the word count, assuming LAPACK's column-major layout.

Next, we consider the Householder-based approach of Murata and Horikoshi [1975], given as $\mathrm{MH}$ in the second row of Table III. In this algorithm, each column is eliminated all at once, and the bulge is chased completely off the band before the next column is eliminated. Because of operations on the triangular fill-in, the number of flops required increases to $6 n^{2} b$ compared to Givens-based algorithms. Since each bulge is chased entirely off the band, the entire band must be read from slow memory for every column eliminated, a total of $O\left(n^{2} b\right)$ words moved. Assuming column-major layout, the sequence of bulge chases for each column (i.e., bulges $(j, k)$ for fixed $j$ ) is executed on contiguous data, and the message count is a factor of $O(M)$ less than the word count. 
In order to reduce communication costs for the $\mathrm{MH}$ algorithm it is possible to apply one of the optimizations described in Section 4: chasing multiple bulges. From Lemma 4.4 , we can chase $O\left(M / b^{2}\right)$ bulges at a time and maintain a working set which fits in fast memory. This results in a reduction of both bandwidth and message counts by a factor of $O\left(M / b^{2}\right)$. We call this algorithm "Improved $\mathrm{MH}$," given in the third row of Table III.

Consider an algorithm within the SBR framework with parameters $\left\{\left(b_{i}, c_{i}, d_{i}\right), i=\right.$ $1,2, \ldots, s\}$, which does not chase multiple bulges at a time (i.e., $\omega_{i}=1$ for every $i$ ). This corresponds to the fourth row of Table III. The flop count is given by Bischof et al. [2000b, Equation (3)] (and Lemma 5.6). Note that the approach of Rajamanickam [2009] allows the flop count to be reduced to $4 n^{2} b$ for all parameter choices. Since the SBR framework is right-looking, the trailing band must be read for each parallelogram eliminated. During the $i$ th step, there are $O\left(n / c_{i}\right)$ parallelograms and each parallelogram is chased $O\left(n / b_{i}\right)$ times. The amount of data accessed during one bulge chase is $O\left(b_{i}\left(c_{i}+d_{i}\right)\right)$ words - for example, $b_{i}$ columns are accessed during the left update and each bulge occupies $c_{i}+d_{i}$ rows. Thus, the number of words read during the $i$ th step is $O\left(n^{2}\left(1+d_{i} / c_{i}\right)\right)$. In the best case, the message count is a factor of $M$ smaller than the word count.

If we apply the successive halving approach $\left(c_{i}=d_{i}=b_{i} / 2\right)$ but do not chase multiple bulges, then the counts for SBR simplify to $O\left(n^{2} t\right)$ words (where $t \leq \log b$ is the smallest step index such that $n\left(b_{t}+d_{t}+1\right) \leq M$, or $t=\log b$ otherwise) and $O\left(n^{2} t / M\right)$ messages, in the best case. These counts appear in the fourth row of Table III.

5.1.2. CA-SBR. The communication avoiding sequential algorithm, shown in Algorithm 1, is based on the framework given in Bischof et al. [2000b], using the successive halving approach (see Section 4.3). Our main deviation from the original SBR framework is chasing multiple bulges at a time, as described in Section 4.2. Recall that $\omega_{i}$ denotes the number of bulges chased at a time, and $\ell_{i}$ the number of times each bulge is chased, during step $i$. We would like to maximize $\omega_{i}$ so that for some $\ell_{i} \geq 1$, this working set fits in a fast memory of size $M$ words. We ignore the sparsity below the $b_{i}$ th subdiagonal by assuming each column has $b_{i}+d_{i}+1$ nonzeros (i.e., the working bandwidth). It follows from Lemma 4.4 that we would like to pick positive integers $\omega_{i}$ and $\ell_{i}$ such that $\omega_{i}$ is maximized and

$$
\left(\left(\omega_{i}-1\right)\left(2 b_{i}-c_{i}\right)+c_{i}+d_{i}+b_{i} \ell_{i}\right)\left(b_{i}+d_{i}+1\right) \leq M .
$$

We use a successive halving approach, as mentioned before. That is, at each step $i$, we cut the remaining bandwidth $b_{i}$ in half by setting $d_{i}=b_{i} / 2$. We also set $c_{i}=b_{i} / 2$ (which satisfies Constraint 4.1). To simplify the analysis, we assume that the initial bandwidth $b=b_{1}$ is a power of two. ${ }^{9}$

As in Lemma 4.4, when chasing a set of $\omega_{i}$ bulges, we work right-to-left, chasing each bulge $\ell_{i}=(3 / 2) \omega_{i}$ times in turn. In this way, after all bulges in the set are chased, the set does not overlap the previous columns occupied, and the relative positions of the bulges are maintained. This process is shown in Figure 2 and corresponds to line 9 in Algorithm 1. Fixing $\ell_{i}$ in terms of $\omega_{i}$ also has the benefit of decreasing the message count on successive steps. While the constant ratio between $\omega_{i}$ and $\ell_{i}$ simplifies theoretical analysis, these parameters can be tuned independently in practice.

With these parameter choices and assumptions, Inequality (2) simplifies, as given in the following constraint.

\footnotetext{
${ }^{9}$ We can always pad the starting bandwidth out to a power of 2 and increase our costs by constant factors, that is, not affecting the asymptotic analysis. In practice, using floors/ceilings and careful bookkeeping is sufficient.
} 


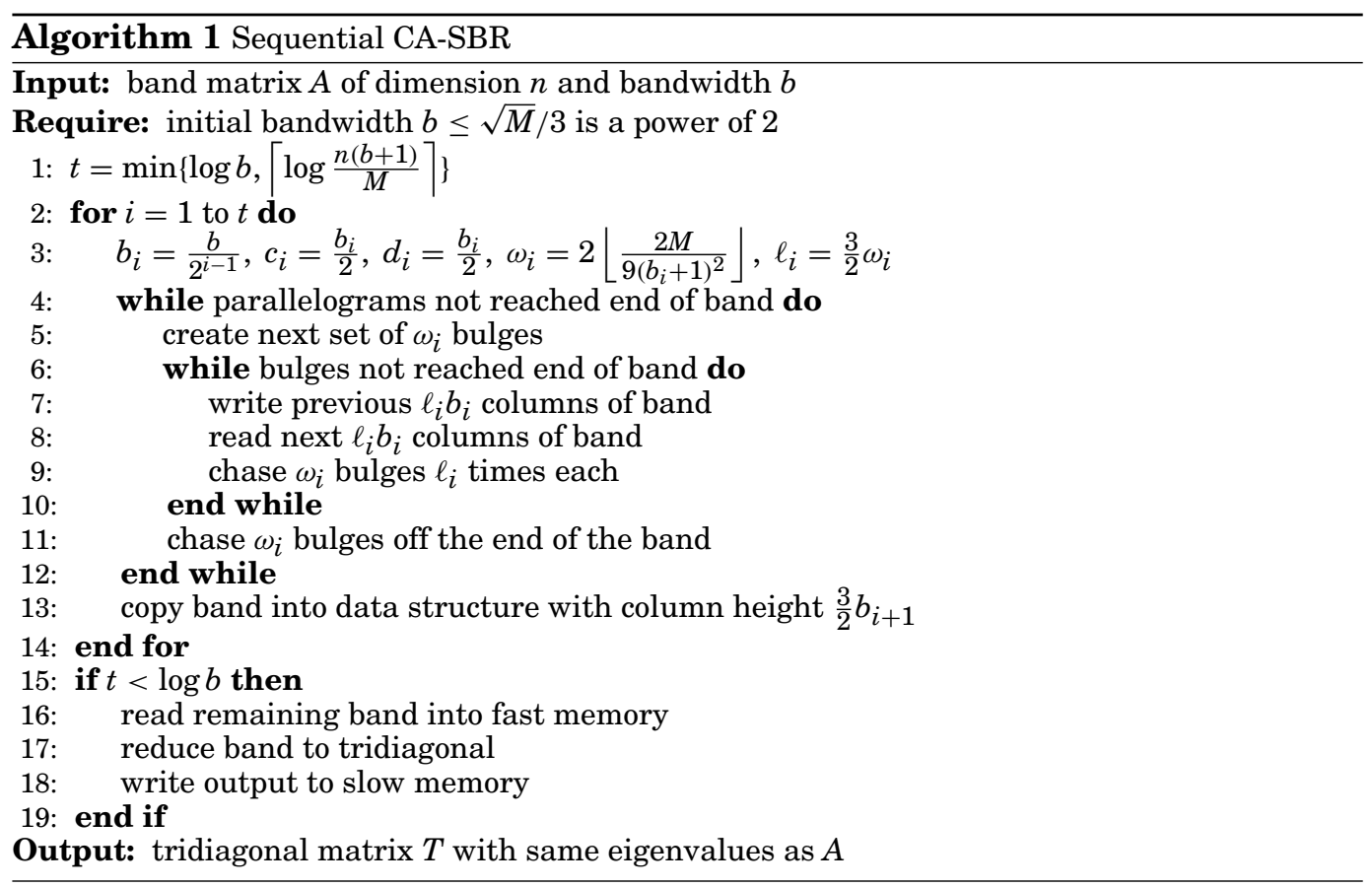

Constraint 5.1. Assuming $b$ and $\omega$ are even, $c=d=b / 2, \ell=(3 / 2) \omega$, and $b \leq$ $\sqrt{M} / 3$, then the number of bulges chased at a time must not exceed $\omega \leq 4 M /\left(9(b+1)^{2}\right)$.

By satisfying Constraint 5.1, we ensure that the entire operation can be performed on columns which all fit in fast memory simultaneously.

We include explicit memory operations within the algorithm in order to determine the communication costs: writes imply moving data from fast memory to slow memory and reads imply moving data from slow memory to fast memory.

We omit the details of creating a set of bulges (line 5) and of chasing bulges at the end of the band (line 11). Both the arithmetic and communication costs of creating $\omega_{i}$ bulges or chasing $\omega_{i}$ bulges off the end of the band are dominated by that of chasing the $\omega_{i}$ bulges $\ell_{i}$ times each. Also, since neither operation occurs in the inner loop of the algorithm, they contribute only lower-order terms to the counts for the entire algorithm.

The computation of $t$ in line 1 determines the step (if any) after which the remaining band fits entirely in fast memory. Note that if $n>M$, then the band will never fit in fast memory and $t=\log b$. If the band becomes small enough to fit in fast memory, then the algorithm will stop the main loop (lines 2-14) and fall to the clean-up code in lines 15-19 which simply reads the band into fast memory, reduces to tridiagonal form, and writes the result back to slow memory.

Flop Count. In order to count the number of flops required by Algorithm 1, we first establish two lemmas related to the cost of applying Householder transformations.

LEMMA 5.2. Applying a Householder transformation from the left, House $(u) \cdot A$, incurs no more than $4 h c+h-c$ flops, where $h$ is the number of nonzeros in $u$ and $A$ has c columns. Equivalently, applying the transformation from the right, $A$. House $(u)$, incurs no more than $4 h r+h-r$ flops if $A$ has $r$ rows. 
PRoOF. The first statement is verified by counting the operations in $A:=A-$ ( $\tau u)\left(u^{T} A\right)$. The second statement is verified by transposing the first transformation. $\square$

LEMMA 5.3. Applying a Householder transformation symmetrically to an $n$-by- $n$ symmetric matrix $A$, House $(u) \cdot A \cdot \operatorname{House}(u)^{T}$, incurs no more than $(4 h-1) n+5 h$ flops, where $h$ is the number of nonzeros in $u$.

PROOF. We perform three steps: $y:=A(\tau u), v:=y-(1 / 2)\left(y^{T} u\right) u$, and $A:=A-$ $u v^{T}-v u^{T}$. The first step incurs $(2 h-1) n+h$ flops, the second $4 h-1$, and the third $2 n h$, if we exploit symmetry.

Given these lemmas, we can compute the flop count of a single bulge chase.

LEMMA 5.4. A single bulge chase requires $8 b c d+4 c d^{2}+O(b c)$ flops. Creating a bulge, or clearing a bulge (off the end of the band), is less expensive.

PROOF. We refer to the four operations depicted in Figure 1 . Let $1 \leq m \leq c$ index the (unblocked) Householder transformations that eliminate the parallelogram in the $\mathrm{QR}$ region. Transformation $m$ is applied from the left to $c-m$ columns in the $\mathrm{QR}$ region and $b-c$ columns in the PRE region, from the right to $b-(c-m)$ rows in the POST region, and symmetrically to a $(d+c)$-by- $(d+c)$ symmetric matrix in the SYM region. Applying Lemmas 5.2 and 5.3, transformation $m$ performs $8 b d+4 d^{2}+O(b)$ flops, and there are $c$ transformations. Creating a bulge is less expensive because the PRE region includes only $b-c-d$ columns. As a result, transformation $m$ does fewer flops. Clearing a bulge is less expensive because there are fewer rows in the POST region.

See Ballard et al. [2012, Section 5.3] for a discussion of different approaches to chasing individual bulges and their implications on performance.

We can also count the number of bulge chases that occur during each step.

LEMMA 5.5. The number of bulges chased during a step with parameters $n, b, c$, and $d$ is $n^{2} /(2 b c)+O(n / b)$.

PROOF. For each parallelogram eliminated, the bulge must be chased the length of the trailing band, in increments of $b$ columns. Thus, the total number of bulge chases during a step is $\sum_{j=1}^{n / c}(n-j c) / b=n^{2} /(2 b c)+O(n / b)$.

Lemmas 5.4 and 5.5 together imply the following fact, which agrees with Bischof et al. [2000b, Equation (3)].

LEMMA 5.6. The flop count of eliminating $d$ diagonals from a matrix with bandwidth $b$ using $S B R$ is $\left(4 d+2 d^{2} / b\right) n^{2}+O\left(n^{2}\right)$.

The order of operations specified by the algorithm does not affect the flop count, provided Constraints 4.1 and 4.2 are satisfied. Given the cost of the $i$ th step specified by Lemma 5.6, since $d_{i}=b_{i} / 2$ and $\sum_{i} d_{i}=b-1$, the flop count of Algorithm 1 (ignoring lower-order terms) is

$$
\sum_{i=1}^{\log b}\left(4 d_{i}+2 \frac{d_{i}^{2}}{b_{i}}\right) n^{2}=5 n^{2} b .
$$

Word Count. In determining the communication costs of Algorithm 1, we must consider two cases. If $n>M$, then $\log b<\lceil\log (n(b+1) / M)\rceil$ and the main loop (lines 2-14) will be executed $\log b$ times, reducing the band to tridiagonal. However, if $n<M$, then at some point the bandwidth will become small enough such that the entire band fits in 
fast memory. At this point, the algorithm reduces to lines 15-19 and the only communication required to finish the reduction is that of reading the band into fast memory and writing the tridiagonal output back to slow memory for $O\left(n b_{t+1}\right)$ words.

We now consider the $i$ th step, where we assume the band is too large to fit in fast memory. The dominant communication cost is in the innermost loop (lines 6-10). There are $(3 / 2) b_{i}$ elements in each column, so one iteration of the inner loop moves $3 \ell_{i} b_{i}^{2}=$ $O(M)$ words. The inner loop is executed $O\left(n /\left(\ell_{i} b_{i}\right)\right)$ times for each set of bulges, and there are $O\left(n /\left(c_{i} \omega_{i}\right)\right)$ sets of bulges during the step. Thus, one step moves $O\left(n^{2} b_{i}^{2} / M\right)$ words.

The number of words moved by Algorithm 1 is then

$$
\sum_{i=1}^{t} O\left(\frac{n^{2} b_{i}^{2}}{M}\right)+O\left(n b_{t+1}\right)=O\left(\frac{n^{2} b^{2}}{M}+n b\right) .
$$

Message Count. We will assume the band matrix is stored in LAPACK symmetric band storage format (column-major with column height equal to the working bandwidth) so that any block of columns of the band will be stored contiguously in slow memory. After each set of subdiagonals is annihilated from a column block, the algorithm packs the remaining diagonals into a smaller data structure (see line 13) to maintain a packed column-major layout for all successive steps. This increases the memory footprint by no more than a factor of two and can also be done in place, and it adds only lower-order terms to the word and message counts.

As in the previous section, if the band becomes small enough to fit in fast memory, then the communication costs of completing the algorithm reduce to those of reading the band and writing the tridiagonal output. In this case, this involves 2 messages. When the band is too large to fit in fast memory, the dominant message count is that of the innermost loop. Since consecutive columns are stored contiguously, each iteration of the innermost loop involves 2 messages. As argued earlier, the inner loop is executed $O\left(n /\left(\ell_{i} b_{i}\right)\right)$ times for each set of bulges, and there are $O\left(n /\left(c_{i} \omega_{i}\right)\right)$ sets of bulges during the step. Thus, the one step involves $O\left(n^{2} b_{i}^{2} / M^{2}\right)$ messages.

The number of messages moved by Algorithm 1 is then

$$
\sum_{i=1}^{t} O\left(\frac{n^{2} b_{i}^{2}}{M^{2}}\right)+O(1)=O\left(\frac{n^{2} b^{2}}{M^{2}}+1\right) .
$$

\subsection{Computing Eigenvalues and Eigenvectors}

In the context of multistep tridiagonalization, we have a sequence $Q_{1}=U_{0} U_{1} \cdots U_{t}$ of orthogonal transformations that reduce the starting matrix to tridiagonal form. Here, $U_{0}$ corresponds the full-to-band step in Section 3 and $U_{1}, \ldots, U_{t}$ correspond to the band reduction steps in this section (we let $U_{0}=I$, an $n$-by- $n$ identity matrix, if starting from banded form). When only eigenvalues are desired, these transformations can be discarded, but when eigenvectors are desired, these transformations must be used to reconstruct the eigenvectors $V=Q_{1} Q_{2}$ of the starting matrix from the eigenvectors $Q_{2}$ of the tridiagonal matrix.

Compared to Section 5.1, the main difference between computing eigenvalues and additionally eigenvectors is that the costs of computing $Q_{1} Q_{2}$ increase with the number of steps taken in the band reduction. For example, while the flop count of the band reduction in Section 5.1 ranges from $4 n^{2} b$ to $6 n^{2} b$, that of the back-transformation ranges from $2 n^{3}$ up to $n^{3} \log b$ (similar logarithmic factors appear in the word and message counts, too). This observation is based on the following result. 
LEMMA 5.7. The flop count of applying all the updates from a single band reduction step to a dense $n$-by-n matrix is $2 \frac{d}{b} n^{3}$, ignoring lower-order terms.

PROOF. From Lemma 5.5, there are $n^{2} /(2 b c)$ bulge chases, each consisting of $c$ Householder vectors of length $d+1$. From Lemma 5.2, the flop count of applying each Householder transformation to an $n$-by- $n$ matrix is $4(d+1) n$, so the total flop count is $4 d n \cdot\left(n^{2} /(2 b)\right)=2(d / b) n^{3}$, ignoring lower-order terms.

The orthogonal matrix $Q_{1}$ can be constructed explicitly by applying the updates from the band reduction, represented by $U_{1}, \ldots, U_{t}$, to the (explicit) matrix $U_{0}$; this is the approach we will take in CA-SBR (Algorithm 2), because it is the simplest to describe. Some flops may be saved when starting from the identity matrix (compared to applying them to a dense matrix, see e.g., Kaufman [2000]), but the entries fill in quickly after one step, and we will ignore this potential savings in our analysis. Then, the flop count of computing $Q_{1} Q_{2}$ given $Q_{2}$ is that of a matrix multiplication, $2 n^{3}$ flops. (Alternatively, if $Q_{2}$ is computed by $Q R$ iteration, then its updates can be applied to $Q_{1}$, yielding $Q_{1} Q_{2}$ without forming $Q_{2}$ explicitly.) Another option is to store $Q_{1}$ implicitly as a set of Householder vectors and apply them to $Q_{2}$. While this choice does not affect our theoretical analysis of CA-SBR, it should be considered in practice, especially in the case of computing a partial eigensystem, where $Q_{2}$ may have many fewer than $n$ columns. As a trade-off, however, storing the Householder information for each step requires extra memory for $\Theta\left(n^{2}\right)$ (at most $n^{2} / 2$ ) entries per step.

Table IV shows the flop, word, and message counts for various approaches to tridiagonalizing a band matrix (for computing both eigenvalues and eigenvectors), as presented and analyzed next in Sections 5.2.1 and 5.2.2.

In the context of multistep tridiagonalization, the communication lower bounds referenced in Section 2.5 apply to the first step (full-to-band), but not the subsequent steps. However, note that a lower bound for part of the algorithm gives a valid lower bound for the whole algorithm. So, we will compare the approaches in Table IV (the subsequent steps) and see which attain the lower bounds of $\Omega\left(n^{3} / \sqrt{M}\right)$ words and $\Omega\left(n^{3} / M^{3 / 2}\right)$ messages; both bounds are attainable in the first step by setting $b=\Theta(\sqrt{M})$. We claim that only CA-SBR attains these expected lower bounds for all ranges of parameters we consider, within a factor of $t=O(\log M)$. In practice, because of the extra costs of back-transformation (proportional to the number of band reduction steps), a hybrid approach may be better. For example, one could use CA-SBR until the bandwidth has been reduced to $b=O\left(n^{1 / 3} M^{1 / 6}\right)$ and then use Improved BLS, which would guarantee $t=O(1)$ and the lower bounds are attained, thus the cost of the back-transformation need not increase asymptotically.

Clearly the counts of LAPACK asymptotically exceed the lower bounds. BLS or improved LAPACK may or may not attain the lower bounds, depending on whether $b M^{1 / 2} / n=O(1)$. In the case of improved BLS, it depends on whether $b^{3} /\left(n M^{1 / 2}\right)=$ $O(1)$. In order to attain the lower bound in the full-to-banded step, we must choose $b=\Theta(\sqrt{M})$. In this case, if $n \ll M$, the word counts of the band reduction for Improved LAPACK, BLS, and Improved BLS asymptotically exceed the lower bound. If $n=\Omega(M)$ and $b=O(\sqrt{M})$, then the word counts of those three approaches match the lower bound, and the message count of Improved BLS also matches the lower bound.

5.2.1. Alternative Algorithms. As mentioned in Section 5.1.1, the current LAPACK reference code for band reduction (sbtrd) is based on Kaufman [2000]. When the eigenvector matrix $W$ of the band matrix is requested, it is represented as $W=U Q_{2}$, where $U$ 
Table IV.

We compare previous sequential algorithms for tridiagonalization (for eigenvalues and eigenvectors) with our improvements, for symmetric band matrices of $n$ columns and $b+1$ subdiagonals on a machine with fast memory of size $M$. We include the cost of the back transformation (but not the cost of the tridiagonal eigendecomposition). The table assumes that $n b \gg M$ and that $2 \leq b \leq \sqrt{M} / 3$. The two terms summed in the communication costs correspond to the band reduction and back transformation, respectively. In the last row, $t=O(\min \{\log b, \log (n b / M)\})$.

\begin{tabular}{|c|c|c|c|}
\hline Algorithm & Flops & Words & Messages \\
\hline LAPACK [Kaufman 2000] & $2 n^{3}$ & $O\left(n^{2} b+n^{3}\right)$ & $O\left(n^{2} b+\frac{n^{3}}{M}\right)$ \\
\hline Improved LAPACK & $2 n^{3}$ & $O\left(n^{2} b+\frac{n^{3}}{\sqrt{M}}\right)$ & $O\left(n^{2} b+\frac{n^{3}}{M}\right)$ \\
\hline BLS [Bischof et al. 1994] & $2 n^{3}$ & $O\left(n^{2} b+\frac{n^{3}}{\sqrt{M}}\right)$ & $O\left(\frac{n^{2} b}{M}+\frac{n^{3}}{M}\right)$ \\
\hline Improved BLS & $2 n^{3}$ & $O\left(\frac{n^{2} b^{3}}{M}+\frac{n^{3}}{\sqrt{M}}\right)$ & $O\left(\frac{n^{2} b^{3}}{M^{2}}+\frac{n^{3}}{M^{3 / 2}}\right)$ \\
\hline CA-SBR & $t n^{3}$ & $O\left(\frac{n^{2} b}{\sqrt{M}}+\frac{t n^{3}}{\sqrt{M}}\right)$ & $O\left(\frac{t n^{2}}{M}+\frac{t n^{3}}{M^{3 / 2}}\right)$ \\
\hline
\end{tabular}

is the orthogonal transformation that tridiagonalized the band matrix and $Q_{2}$ is the tridiagonal eigenvector matrix; the product $U Q_{2}$ can be either explicitly formed or $U$ can be returned in an implicit form, to be applied later. The LAPACK routine for solving the eigenproblem for a band matrix (sbevd) forms $U$ explicitly and premultiplies $Q_{2}$ by it. Forming $U$ requires approximately (4/3) $n^{3}$ flops [Kaufman 2000], and matrix multiplication requires $2 n^{3}$. In Table IV, we do not count computing $U$, because the Givens rotations can be stored, reordered, and later applied to $Q_{2}$ for a total of $2 n^{3}$ flops, although LAPACK does not offer this functionality.

The word and message counts of the band reduction are analyzed in Section 5.1.1. Assuming the stored Givens rotations are applied to the rows of $Q_{2}$ one at a time (which is how they are accumulated in $U$ in LAPACK), at least one of the rows must be read from slow memory, and the data reuse is $O(1)$. This implies that the word count of the band reduction, $O\left(n^{2} b\right)$, is dominated by that of the orthogonal updates. In the best case, if $Q_{2}$ is stored in row-major order and $n>M$, the message count is $O\left(n^{3} / M\right)$.

Bischof et al. [1994] consider an alternative approach for computing both eigenvalues and eigenvectors of a band matrix, in the context of a two-step reduction of a full symmetric matrix (i.e., $Q_{1}=U_{0} U_{1}$ ). The second (band-to-tridiagonal) step follows the algorithm of Murata and Horikoshi [1975], using $d=b-1$ and $c=1$. The key idea from Bischof et al. [1994] is to store all of the Householder vectors comprising $U_{1}$ and, instead of applying them to $Q_{2}$ in exactly the reverse order that they were computed, to use a reordering technique that respects the dependency pattern. This reordering allows for the orthogonal updates to be blocked (they also show that the updates $U_{0}$ from the full-to-band step can be applied efficiently from an implicit representation, but the dependency pattern is much simpler). See Figure 2 in Bischof et al. [1994] or Figure 2 in Auckenthaler et al. [2011a] for illustrations of this technique. Since the band reduction is performed in one step, the flop count is $2 n^{3}$. Using the reordering technique with a blocking factor of size $\Theta(\sqrt{M})$, the word count of the orthogonal updates is $O\left(n^{3} / \sqrt{M}\right)$. While the orthogonal updates are performed efficiently, the data reuse obtained during the band reduction is $O(1)$, as explained in Section 5.1.1. Thus, the word count of the band reduction is $O\left(n^{2} b\right)$ which dominates the total word count for $b \gg n / \sqrt{M}$. In the best case, the message count of the band reduction is $O\left(n^{2} b / M\right)$. In order to determine the message count of the orthogonal updates, we 
assume the matrix $Q_{2}$ is stored in column-major order and the Householder vectors are written to memory in the order they are computed. Then every application of a block of Householder vectors involves $O(\sqrt{M})$ messages, and so the message count is a factor of $O(\sqrt{M})$ less than the word count. We refer to this as BLS, given in the third row of Table IV.

Note that this same reordering optimization from Bischof et al. [1994] can be used to improve the LAPACK algorithm. That is, the Givens rotations may be reordered and applied to $Q_{2}$ in a blocked fashion. For examples of implementations for applying blocks of Givens rotations, see Rajamanickam [2009] and Van Zee et al. [2013]. If the right block size is chosen, the word count of the orthogonal updates can be reduced to $O\left(n^{3} / \sqrt{M}\right)$. We refer to this algorithm as "Improved LAPACK," given in the second row of Table IV. Because of better alternatives, we do not discuss improvements in the message count.

We can apply two optimizations to reduce the communication costs of the BLS algorithm. First, as noted in Section 5.1.1, when $b \ll \sqrt{M}$, the communication costs of the band reduction can be improved by chasing $O\left(M / b^{2}\right)$ bulges at a time, reducing both the word and message counts by factors of $O\left(M / b^{2}\right)$.

Second, we can reduce the message count in performing the orthogonal updates by storing the eigenvector matrix $Q_{2}$ in a block-contiguous layout with block size $C$-by- $C$ with $C=\Theta(\sqrt{M})$ and by performing a data layout transformation of the temporary data structure of Householder vectors. In order to minimize word count, the Householder vectors corresponding to eliminating $\Theta(\sqrt{M})$ columns and chasing their respective bulges off the band should be temporarily stored before accumulating them into the implicit representation of $U_{1}$.

In order to analyze the data layout transformation, we need to consider the temporary storage of Householder vectors. If we let $H$ be the temporary storage matrix, then we can store each Householder vector associated with the same eliminated column of $A$ in the same column of $H$. Further, each vector can occupy the rows of $H$ corresponding to the rows of $A$ it updated; in this way, $H$ is an $n$-by- $n$ lower triangular matrix. If one bulge is chased at a time and Householder vectors are written to $H$ in the order they are computed, then $H$ will have a column-major data layout. However, in order to improve data reuse in applying the vectors to $Q_{2}$, we want to apply parallelograms of vectors at a time, so we need those parallelograms to be stored contiguously. The data layout transformation is equivalent to transforming a matrix in column-major layout to a block-contiguous layout. By applying (for example) the Separate function given as Algorithm 3 in Ballard et al. [2013b] to each panel of width $\Theta(\sqrt{M})$ a logarithmic number of times, we can convert $H$ from column-major to $\Theta(\sqrt{M})$-by- $\Theta(\sqrt{M})$ blockcontiguous layout with a total of $O\left(n^{2} \log (n / \sqrt{M})\right)$ words and $O\left(\left(n^{2} / M\right) \log (n / \sqrt{M})\right)$ messages, which are lower-order terms for $n \gg \sqrt{M}$.

Note that these two optimizations cannot both be applied straightforwardly to the approach of Bischof et al. [1994], as $H$ will not be written in column-major order when multiple bulges are chased at a time. We claim that a more complicated data layout transformation is possible in the case that multiple bulges are chased at a time. The counts for this algorithm are given as "Improved BLS" in the fourth row of Table IV. We also claim it is possible to apply the second optimization to the LAPACK algorithm, though the order in which the Givens rotations are computed and the method for temporarily storing them is more complicated.

5.2.2. CA-SBR. Algorithm 2 is a modification of Algorithm 1 which includes the explicit formation of the matrix $Q_{1}=U_{0} U_{1} \cdots U_{t}$, which we store in a block-contiguous 
layout with $C$-by- $C$ blocks. As mentioned before, we compute $Q_{1}$ by a sequence of updates to the (explicit) matrix $U_{0}$, the transformation from the full-to-band step (if any, otherwise $U_{0}=I$ ); in our algorithm, we denote the intermediate matrices all as $Q$, starting with $Q=U_{0}$ and ending with $Q=Q_{1}$. Then we compute $V=Q_{1} Q_{2}$ by matrix multiplication. We recall from the previous discussion that we could also store $Q_{1}$ implicitly, for instance, by storing the Householder vectors comprising $U_{0}, U_{1}, \ldots, U_{t}$, and there are scenarios, like partial eigensystems, where the increased storage cost is worthwhile.

An important difference with the case of computing only eigenvalues is the definition of $\omega_{i}$, the number of bulges chased at a time. In Algorithm 1, $\omega_{i}$ is maximized under the constraint that the working set of data to chase $\omega_{i}$ bulges $\ell_{i}$ times each remains of size $O(M)$. In Algorithm 2, $\omega_{i}$ is further limited so that the working set of data while applying the Householder updates to a block row of the intermediate $Q$ matrix remains of size $O(M)$. This working set of data now consists of three components: a subset of $A$, Householder transformations (temporarily stored in a data structure $\mathcal{H}$ ), and blocks of $Q$. We will pick $\omega_{i}$ to be approximately the square root of the previous choice so that each of these three components occupies no more than a third of fast memory. Reducing $\omega_{i}$ results in more communication during the band reduction, but we will see that it is always dominated by that of the orthogonal updates. One advantage of this approach is that, assuming the band is too large to fit into fast memory, Householder information is never written to slow memory: it is computed in fast memory, all updates are applied, and then the Householder entries are discarded.

In order to validate the communication pattern described in Algorithm 2, we verify three facts: $2 \ell_{i} b_{i}$ columns of $A$ fit in one third of fast memory, $\mathcal{H}$ fits in one third of fast memory, and each iteration in the ORTHOGONALUPDATES function involves at most 3 blocks of $Q$, which fit in one third of fast memory. We will show that this is possible when $\omega_{i} \leq 2 \sqrt{M} /\left(9\left(b_{i}+1\right)\right), \ell_{i}=(3 / 2) \omega_{i}, C=\sqrt{M} / 3$, and the given assumption that $b \leq \sqrt{M} / 3$.

Since each column of the band has at most $(3 / 2) b_{i}+1$ entries, the total number of entries in $2 \ell_{i} b_{i}$ columns is $\omega_{i}\left((9 / 2) b_{i}^{2}+3 b_{i}\right)<M / 3$. The $\mathcal{H}$ data structure needs to store Householder information corresponding to chasing $\omega_{i}$ bulges $\ell_{i}$ times each, and each bulge consists of $c_{i}\left(d_{i}+1\right)$ entries. Thus $\mathcal{H}$ requires $(3 / 8) \omega_{i}^{2}\left(b_{i}^{2}+2 b_{i}\right)<M / 3$ words of storage. Finally, we must also verify that the number of columns of $Q$ updated by the $\omega_{i} \ell_{i}$ bulge chases (which correspond to the rows of the band that are updated) cannot span more than 3 blocks of $Q$ (i.e., one third of fast memory). By Lemma 4.3, the number of columns is $(3 / 2) \omega_{i}\left(b_{i}+1\right)-b_{i} / 2 \leq 2 \sqrt{M} / 3$; since $C=\sqrt{M} / 3$, these columns cannot span more than 3 blocks.

Note that $t$ is defined differently here than in Section 5.1.2. Here, since we will eliminate all subdiagonals at once, we need twice the working bandwidth to fit into fast memory.

Flop Count. From Lemma 5.7, the flop count of the orthogonal updates is given by $2 n^{3} \sum_{i=1}^{t} d_{i} / b_{i}$, where $t$ is the number of steps, and the flop count of the band reduction is always a lower-order term. By the definition of $t$ and the fact that $d_{i}=b_{i} / 2$, the flop count is then $n^{3} \min \{\log b,\lceil\log (2 n(b+1) / M)\rceil\}$, ignoring lower-order terms.

Word Count. The word count can be computed in a similar way to Section 5.1.2, though $\omega_{i}$ is defined slightly differently. The dominant communication cost is the call to the function ORTHOGONALUPDATES within the innermost loop (lines 7-12). During the $i$ th step, the number of sets of $\omega_{i}$ bulges is $n /\left(c_{i} \omega_{i}\right)$, and for each set, the innermost loop is executed $O\left(n /\left(\ell_{i} b_{i}\right)\right)$ times. Since $\mathcal{H}$ resides in fast memory, the word count of 


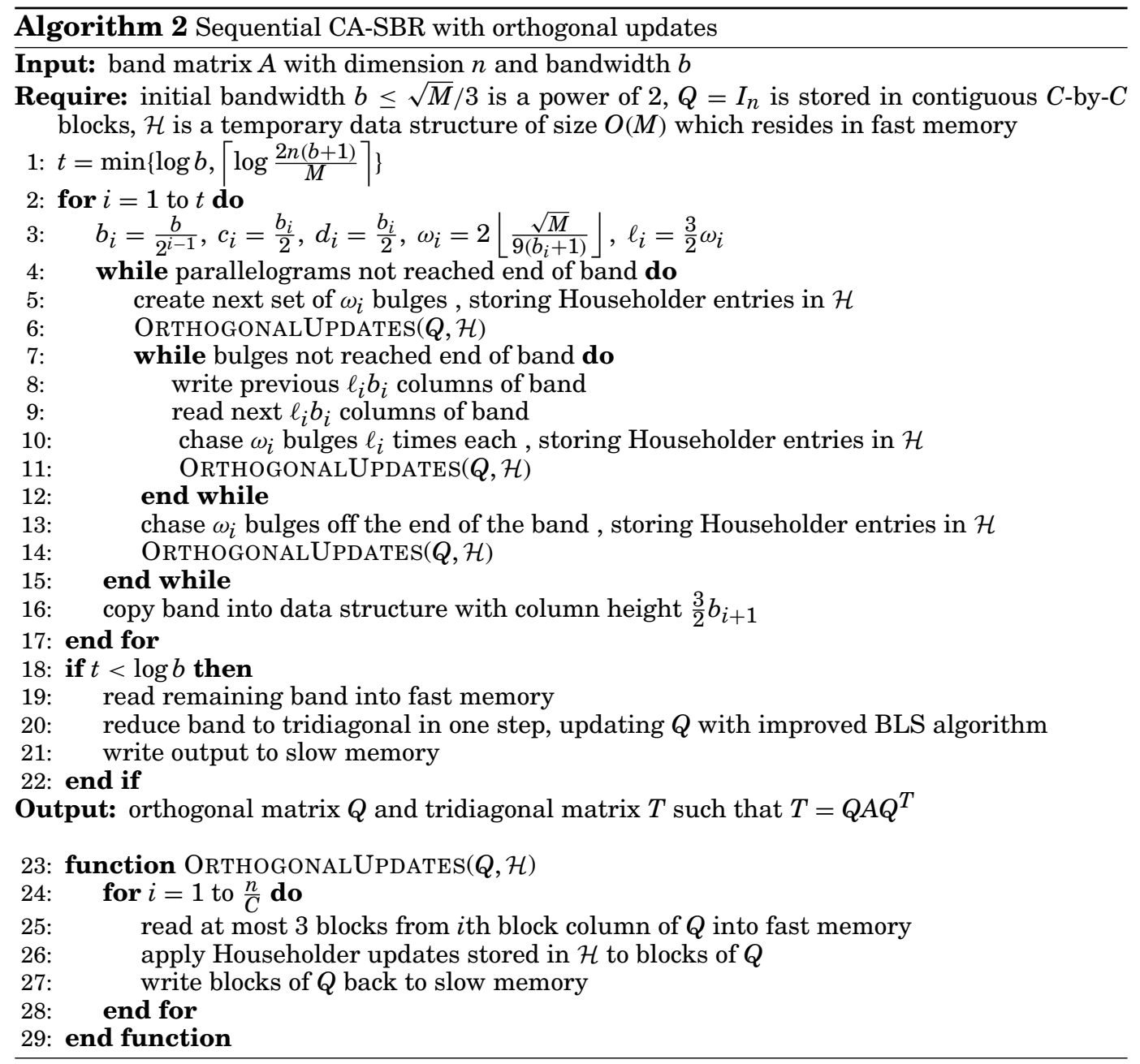

the function ORTHOGONALUPDATES is that of reading and writing the row panels of the $Q$ matrix: $O(n C)$ words. Thus, the total word count of Algorithm 2 is

$$
\sum_{i=0}^{t} O\left(\frac{n^{3}}{\sqrt{M}}\right)=O\left(\frac{t n^{3}}{\sqrt{M}}\right) .
$$

Note that due to the change in definition of $\omega_{i}$, the word count of the band reduction is increased from $O\left(n^{2} b^{2} / M\right)$ (from Section 5.1.2) to $O\left(n^{2} b / \sqrt{M}\right)$, but this higher count is still dominated by that of the orthogonal updates.

In the case that $\lceil\log (2 n(b+1) / M)\rceil<\log b$, the final step of the algorithm is to read the entire band into memory and reduce all the remaining subdiagonals at once, updating $Q$ using the second technique of improving the BLS algorithm (i.e., transforming the column-major $H$ matrix to block-contiguous layout). In this case, the word count of reading $A$ is $O(n b)$, and the word count of the orthogonal updates is $O\left(n^{3} / \sqrt{M}\right)$ as explained before. Both of these are lower-order terms.

Message Count. The message count is also dominated by that of the orthogonal updates. Since $Q$ is stored in $C$-by- $C$ contiguous blocks, the message count of the function 
Table V.

We compare previous parallel algorithms for tridiagonalization (for eigenvalues only) with our improvements, for symmetric band matrices of $n$ columns and $b+1$ subdiagonals on a machine with $p$ processors. The first row assumes that $p \leq n / b$, and the second row assumes $p \leq n /(3 b)$. The asymptotic arithmetic and communication costs are determined along the critical path.

\begin{tabular}{|c|c|c|c|}
\hline Algorithm & Flops & Words & Messages \\
\hline Lang [Lang 1993; Auckenthaler 2012] & $O\left(\frac{n^{2} b}{p}\right)$ & $O(n b)$ & $O(n)$ \\
\hline CA-SBR & $O\left(\frac{n^{2} b}{p}\right)$ & $O(n b)$ & $O(p \log b)$ \\
\hline
\end{tabular}

ORthogonalUpdates is $O(n / C)$. Thus, the message count of Algorithm 2 simplifies to $O\left(\operatorname{tn}^{3} / M^{3 / 2}\right)$.

Like the word count, the message count associated with the band reduction is increased by the choice of $\omega_{i}$, but this higher count of $O\left(t n^{2} / M\right)$ is still dominated by that of the orthogonal updates. In the case that $\lceil\log (n b / M)\rceil<\log b$, the final step of the algorithm using the improved BLS technique incurs a message count which is also a lower-order term.

\section{PARALLEL BAND TRIDIAGONALIZATION ALGORITHMS}

Recall our distributed-memory parallel model, where we have $p$ processors connected over a network. Again, we will first discuss the case of computing eigenvalues only and then extend to the case of computing both eigenvalues and eigenvectors. The main improvement of our new algorithm over previous approaches is a reduction in message count, both in terms of the band reduction and the back-transformation phase (when eigenvectors are desired).

We assume that $b \leq n /(3 p)$, where $p$ is the number of processors involved in the band reduction. This is a reasonable assumption after the first step in a multistep tridiagonalization approach, starting from a full matrix (see Section 3), in order to minimize the message count in the first step. Similarly to the sequential case, we do not treat the case when the input matrix has an intermediate bandwidth $n /(3 p)<b<$ $n-1$; in practice, one may use fewer processors on the first step(s), or have multiple processors participate in a single bulge chase, although the latter approach may incur a higher communication cost - see [Lang 1993].

\subsection{Eigenvalues Only}

In this section we concern ourselves with the case when only eigenvalues are desired, so the orthogonal updates may be discarded after applying them to the band. We assume each of the $p$ processors has $M=\Omega(n b / p)$ words of memory available, so that the band $A$ can be stored across the machine. We collect the results from the analyses in Sections 6.1.1-6.1.2 in Table V.

6.1.1. Alternate Approaches. The "conventional" distributed memory band tridiagonalization algorithm was introduced in Lang [1993], and has been extended several times. This is a parallelization of the MH algorithm, discussed in Section 5.1.1, a one-step band reduction algorithm (i.e., $d=b-1$ and $c=1$ ). We will refer to this as Lang's algorithm.

For brevity, we will not present this algorithm and its variants, but instead refer the reader to the detailed complexity analysis (and performance modeling) in Auckenthaler [2012] (summarized in Auckenthaler et al. [2011a] and Auckenthaler et al. [2011b]). We present their complexity results in asymptotic notation; the hidden constant factors vary depending on the optimizations applied, including "logical blocking," which eliminates a factor of 2 idle time along the critical path, and using a cyclic 


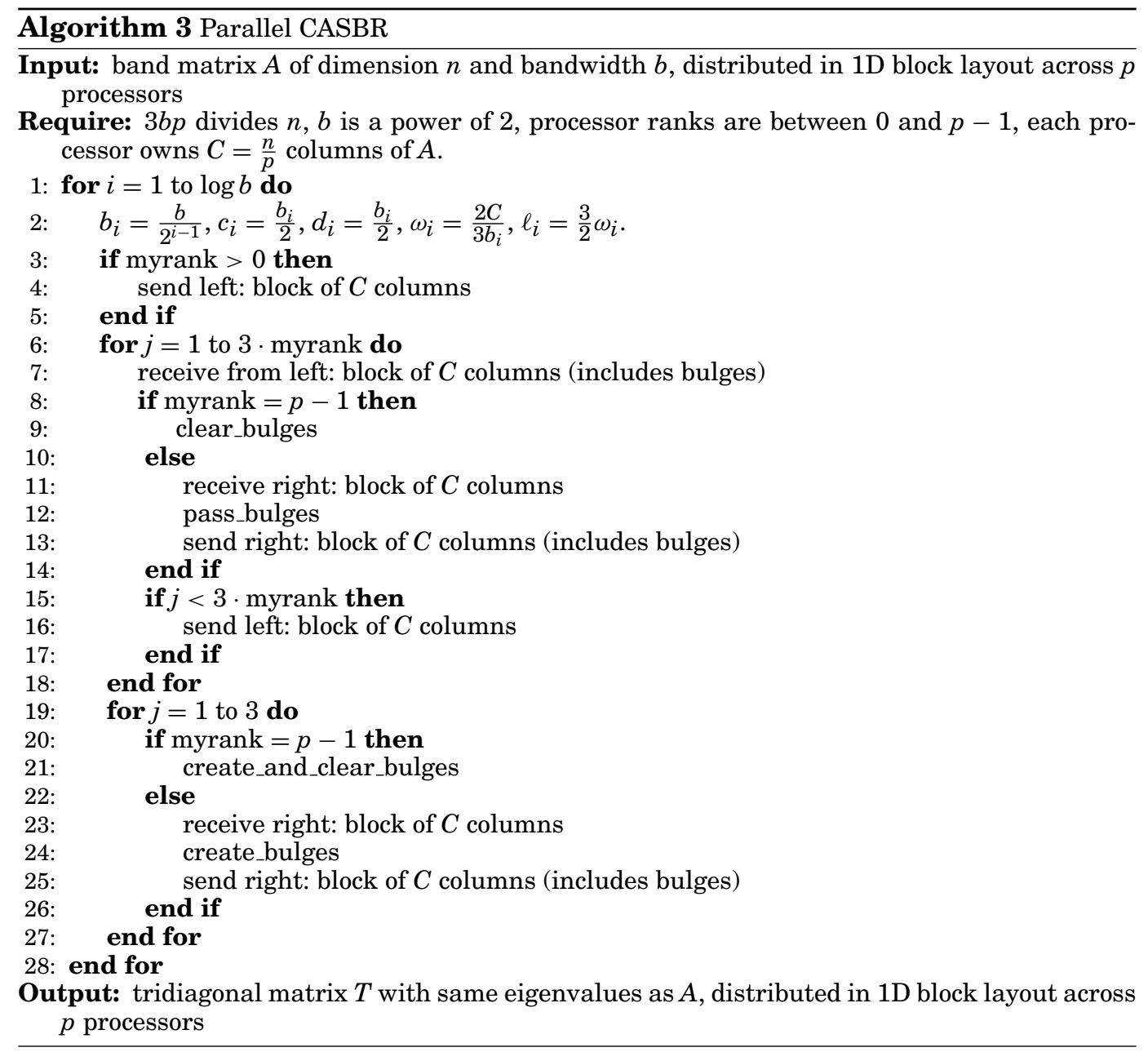

layout, which helps alleviate load imbalance between processors. Along the critical path, their algorithm performs $O\left(n^{2} b / p\right)$ flops and moves $O(n b)$ words. Because there is a communication step for every column in the band, the message count is $O(n)$.

Unless multiple bulges are chased at a time, the message count of $O(n)$ cannot be asymptotically reduced. That is, if a message is sent along the critical path for every parallelogram annihilated, then the last step, which has one parallelogram for each column, will incur $O(n)$ messages.

6.1.2. CA-SBR. The parallel CA-SBR algorithm (Algorithm 3) begins with a similar data layout as Lang's algorithm. Each of the $p$ processors (indexed 0 to $p-1$ ) owns a contiguous set of $C=n / p$ columns of the lower half of the symmetric band. We use a similar successive-halving and multiple-bulge-chasing approach to the sequential CA-SBR algorithm. During each step, the number of columns per processor stays fixed at $C=n / p$. To simplify the presentation, we assume that $3 b p$ divides $n$, and that $b$ is a power of 2 . This implies that $p \leq n /(3 b)$, which is our maximum parallelism. These constant factors will not affect our asymptotic analysis.

Roughly, the parallel algorithm proceeds as each processor chases bulges through its $C$ (local) columns and into the $C$ columns of its right neighbor, and then passes 
the second set of columns to its right neighbor. This way, each of the $p$ processors accesses only $O(n b / p)$ of $A$ rather than streaming through the entire band. Figure 3 shows the generic pattern of computation and communication within the algorithm. In the algorithm we present below, each processor is active on every other step; we can eliminate this idle time by using logical blocking (as in Lang [1993]); we ignore this factor of 2 savings for the purposes of our asymptotic analysis.

At the high level, there are four local computational kernels: create_bulges, pass_bulges, clear_bulges, and create_and_clear_bulges. The create_bulges kernel eliminates $\omega_{i}$ parallelograms (each with $c_{i}$ columns and $d_{i}$ diagonals) from the local set of $C$ columns of $A$ and chases the resulting bulges $\ell_{i}$ times (on average ${ }^{10}$ ) into the right neighbor's set of $C$ columns. The pass_bulges kernel chases $\omega_{i}$ bulges (created by the left neighbor) from the local set $\ell_{i}$ times into the right neighbor's set. The create_and_clear_bulges and clear_bulges kernels are only executed by the last processor ${ }^{11}$ and are analogous to create_bulges and pass_bulges, except the 'second set of columns' is off the end of the band. Both create_bulges and pass_bulges require $2 C$ columns to pass information from one processor's columns to the next: the left set of $C$ columns is owned by the processor invoking the kernel, and the right set is owned by the right neighbor. The create_and_clear_bulges and clear_bulges kernels require only the last $C$ columns of the band (its local set).

At any time, a processor will have access to and update only its own $C$ columns and the $C$ columns from its right neighbor. For example, the parallel algorithm begins with processor 1 sending its columns to processor 0 . After processor 0 executes the create_bulges kernel, it sends the updated $2^{\text {nd }}$ set of $C$ columns (with bulges) back to processor 1. Processor 1 must then also receive processor 2's $C$ columns in order to execute the pass_bulges kernel. The parallel algorithm ends (on step $i=\log b$ ) with processor $p-1$ receiving $C$ columns from the left, clearing all bulges, and finally eliminating the last subdiagonal of its local block (via create_and_clear_bulges).

In order for the pass_bulges kernel to pass the bulges into the right neighbor's column block, and for the bulges to retain their respective positions relative to the column blocks, we set $\ell_{i}=C / b_{i}$, which is an integer given the previous assumptions. Recall that a bulge chase advances a bulge exactly $b_{i}$ columns.

Our constraint on $\omega_{i}$, the maximum number of bulges that fits in $C=n / p$ columns, is given by Lemma 4.4:

$$
\left(\omega_{i}-1\right)\left(2 b_{i}-c_{i}\right)+c_{i}+d_{i} \leq C .
$$

A little more care must be taken when creating bulges to ensure that they do not cross processor boundaries (adjacent sets of $C$ columns). Consulting Lemma 4.3, for the successive halving approach, we arrive at the following lemma.

Lemma 6.1. Assuming $b$ and $\omega$ are even, $c=d=b / 2$, and $3 b$ divides $C$, then we can create and chase $\omega=2 C /(3 b)$ bulges at a time, and chasing them $\ell=C / b$ times each advances them to the next set of $C$ columns.

As in the sequential case, we fix the parameters to simplify the asymptotic analysis; in practice, the parameters (including the number of processors $p^{\prime} \leq p$ used and the number of columns $C$ a processor owns) should be tuned independently.

We analyze the arithmetic, bandwidth, and message counts along the critical path of the algorithm. That is, we follow the progress of the first $\omega_{1}$ bulges from processor 0

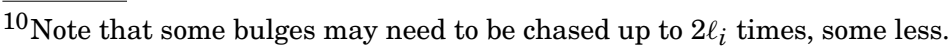

${ }^{11}$ Note that processor $p-2$ may chase some bulges (partially or completely) off the end of the band when invoking pass_bulges and create_bulges, depending on the number of columns owned by processor $p-1$ and the current bandwidth.
} 


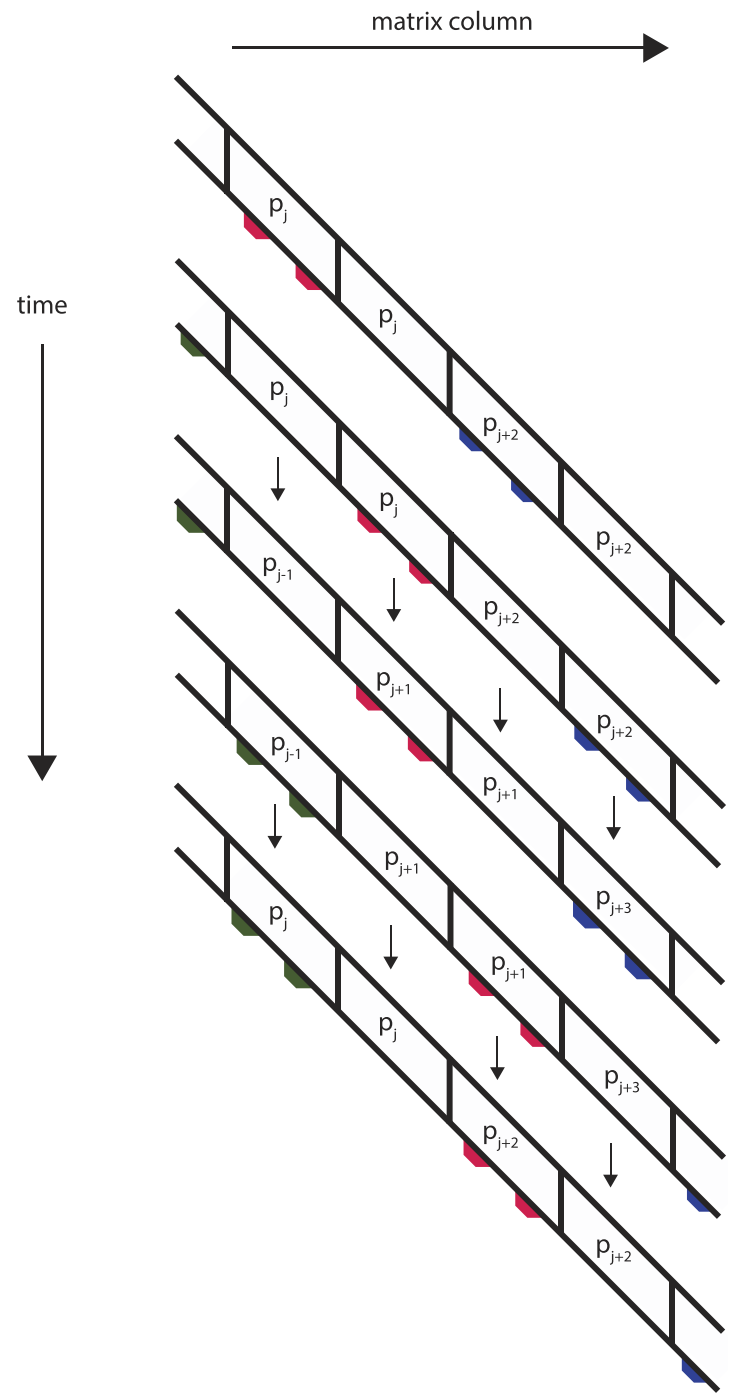

Fig. 3. Generic computation/communication pattern in the course of parallel CA-SBR (band reduction only). Time progresses down the y-axis, and a section of the band matrix is shown for a sequence of steps, with each matrix column remaining fixed on the x-axis. Labels on block columns specify which processor owns the block at the time step, and bulge colors indicate common pairs of parallelogram indices. The transition between first and second steps corresponds to computation, and then the transitions alternate between communication (denoted by arrows) and computation.

to processor $p-2$, at which point (exactly) one of processors $p-2$ and $p-1$ is active chasing and/or clearing bulges on every remaining step of every step.

Flop Count. From Lemma 5.4, the flop count of chasing one bulge (a single hop), with parameters $b, c$, and $d$, is bounded above by $8 b c d+4 c d^{2}+O(b c)$ flops, while those of creating a bulge and chasing a bulge partially or completely off the band are less. For our choices $b / 2^{i}=b_{i} / 2=c_{i}=d_{i}$, this is $(5 / 2) b_{i}^{3}$ flops.

Every kernel call involves at most $\omega_{i}$ bulges; the calls to create_bulges, pass_bulges, clear_bulges, and create_and_clear_bulges each chase the bulges about $\ell_{i}$ times, so each 
kernel invocation requires about $\omega_{i} \ell_{i}\left(5 b_{i}^{3} / 2\right)=O\left(n^{2} b_{i} / p^{2}\right)$ flops. Following the critical path, there are (fewer than) $p$ kernel invocations while the pipeline fills. At this point, processors $p-2$ and $p-1$ are active for the remainder of the execution, each invoking a kernel on alternating steps. There are $3 p$ steps (iterations of the inner two for-loops) per step, each with one kernel invocation (along the critical path). Altogether, this is

$$
O\left(\frac{n^{2} b_{1}}{p}\right)+\sum_{i=1}^{\log b} O\left(\frac{n^{2} b_{i}}{p}\right)=O\left(\frac{n^{2} b}{p}\right)
$$

flops. The hidden leading constant is about 20; a cyclic layout and logical blocking as in Lang [1993] can be applied here to reduce this constant to between 5 and 10 (note these same strategies reduced the corresponding constant in Lang's algorithm's flop count from 24 to between 6 and 12).

Word Count. Every message in the algorithm consists of $C$ columns of the band; because of bulges and triangular fill-in stored below the $b_{i}$ th subdiagonal, each message (during the $i$ th step) has size (at most) $C\left(3 b_{i} / 2+1\right)=O\left(n b_{i} / p\right)$ words. Following the critical path as before, we have the upper bound of

$$
O\left(n b_{1}\right)+\sum_{i=1}^{\log b} O\left(n b_{i}\right)=O(n b)
$$

words moved

Message Count. The message count analysis is similar to the word count analysis, replacing the $O\left(n b_{i}\right)$ terms by $O(1)$; in total, we have $O(p \log b)$ messages. This is asymptotically smaller than the $O(n)$ messages that Lang's algorithm sends: we save a factor of $O(n /(p \log b))$ messages.

\subsection{Eigenvalues and Eigenvectors}

We recall the notation from Section 5.2, where we compute $Q_{1}=U_{0} U_{1} \cdots U_{t}$, where $U_{0}$ is the transformation from the full-to-band step (or is an identity matrix if starting from banded form), and $U_{1}, \ldots, U_{t}$ are the transformations from the $t$ steps of the algorithms in this section. Our task is to ultimately compute the eigenvector matrix $V=Q_{1} Q_{2}$, where $Q_{2}$ is the tridiagonal eigenvector matrix.

We assume that $Q_{2}$ is distributed in a $2 \mathrm{D}$ blocked fashion to all $p$ processors, and that the bandwidth $b$ of $A$ is (at most) $1 / 3$ of the width of a block row of $Q_{2}$, that is, $b \leq n /(3 \sqrt{p})$. This means that we will use only $\sqrt{p}$ of the $p$ available processors to perform the band reduction, and all $p$ for the back-transformation. So, we must assume each processor has $M=\Omega\left(n^{2} / p\right)$ words of local memory.

We collect the results from the analyses in Sections 6.2.1-6.2.2 in Table VI. Under our assumptions, for both algorithms, the arithmetic and word counts of the backtransformation always dominate those of the band reduction. The asymptotic flop counts decrease linearly (in $p$ ) as expected. The first step of multistep tridiagonalization can attain the communication lower bounds for parallel dense linear algebra (without extra memory), that is, $\Omega\left(n^{2} / \sqrt{p}\right)$ words moved and $\Omega(\sqrt{p})$ messages, if $b=\Theta(n / \sqrt{p})$; see Section 3. Asymptotically, both algorithms attain the lower bound on words moved, up to a factor of $\Theta(\log (n / \sqrt{p}))$ in the case of CA-SBR (Algorithm 4). However, only CA-SBR attains the latency lower bound of $\Omega(\sqrt{p})$, again up to a factor of $\Theta(\log (n / \sqrt{p}))$.

6.2.1. Alternate Approaches. The approach in Auckenthaler et al. [2011a] stores $Q_{1}$ implicitly (as a sequence of Householder transformations) and then applies $Q_{1}$ to $Q_{2}$ in 
Table VI.

We compare previous parallel algorithms for tridiagonalization (for eigenvalues and eigenvectors) with our improvements, for symmetric band matrices of $n$ columns and $b+1$ subdiagonals on a machine with $p$ processors. We include the cost of the back transformation (but not the cost of the tridiagonal eigendecomposition). The first row assumes $\sqrt{p} \leq n / b$, and the second row assumes $\sqrt{p} \leq n /(3 b)$. The asymptotic arithmetic and communication costs are determined along the critical path. The two terms summed in each cost correspond to the band reduction and the back transformation, respectively.

\begin{tabular}{|c|c|c|c|}
\hline Algorithm & Flops & Words & Messages \\
\hline Lang [Auckenthaler 2012] & $O\left(\frac{n^{2} b}{\sqrt{p}}+\frac{n^{3}}{p}\right)$ & $O\left(n b+\frac{n^{2}}{\sqrt{p}}\right)$ & $O\left(n+\frac{n}{b}\right)$ \\
\hline CA-SBR & $O\left(\frac{n^{2} b}{\sqrt{p}}+\frac{n^{3}}{p} \log b\right)$ & $O\left(n b+\frac{n^{2}}{\sqrt{p}} \log b\right)$ & $O(\sqrt{p} \log b+\sqrt{p} \log b)$ \\
\hline
\end{tabular}

a blocked fashion. The authors give three algorithms to compute $Q_{1} Q_{2}$, with different parallel layouts of the matrix $Q_{2}$ - we consider only their best approach, based on a $2 \mathrm{D}$ layout which is dynamically rebalanced. Before computing $Q_{1} Q_{2}$, we assume each processor owns a $(n / \sqrt{p})$-by- $(n / \sqrt{p})$ block of $Q_{2}$. Again, we refer the reader to the detailed analysis in Auckenthaler [2012]. Along the critical path, the additional counts for the back-transformation are $O\left(n^{3} / p\right)$ flops, $O\left(n^{2} / \sqrt{p}\right)$ words moved, and $O(n / b)$ messages.

6.2.2. CA-SBR. As in the sequential case (Sections 5.2 and 5.2.2), we construct $Q_{1}$ explicitly rather than storing it implicitly. The extra cost of the matrix multiplication $Q_{1} Q_{2}$ is dominated by the cost of constructing $Q_{1}$ and thus will not affect our asymptotic analysis. Again, in practice, this cost can be avoided by storing and applying $Q_{1}$ to $Q_{2}$ as a sequence of Householder transformations, although at the cost of additional memory requirements.

By the assumption $\sqrt{p} \leq n /(3 b)$, we can involve all $\sqrt{p}$ processors in each processor row in a band reduction. Since the flop count for the band reduction is a lower-order term, we can afford to perform the band reduction $\sqrt{p}$ times redundantly (or once, but only on a subset of $\sqrt{p}$ processors). We distribute the band $A$ to each row of the given $\sqrt{p}$-by- $\sqrt{p}$ processor grid; each row performs the band reduction once. Note that each processor owns $C=n / \sqrt{p}$ columns of $A$, rather than $n / p$ (as before).

We use Algorithm 4, a modification of Algorithm 3, which simultaneously computes the band reduction and the $n$-by- $n$ matrix $Q_{1}$. As in the sequential case (Algorithm 2 ), we compute $Q_{1}=U_{0} U_{1} \cdots U_{t}$ by applying orthogonal updates to an intermediate matrix $Q$, where initially $Q=U_{0}$; also as in the sequential case, to simplify the presentation, we again refer to these intermediate matrices all as $Q$, and the intermediate band matrices all as $A$. These orthogonal updates combine columns of $Q$ (but not rows); thus, each processor row may work independently. Each processor row is assigned $C$ contiguous rows of $Q$; the columns of this block row are distributed according to the distribution of the band matrix. That is, if processor $i$ (indexed within a given processor row) owns the first element of the $j$ th row of $A$, then processor $i$ will own the $j$ th column of the corresponding block row of $Q$. In this way, the communication pattern of the blocks of $Q$ between neighboring processors will exactly match the communication pattern of the blocks of the band. Whenever a processor performs a local kernel on $2 C$ columns of the band, it will also apply all of those updates to $2 C$ columns of (its block row of) $Q$. This implies that in step $i$, within each processor row, the first processor owns the first $C+b_{i}$ columns of the corresponding block row of $Q$, each subsequent processor owns the next $C$ columns, and the last processor owns the last $C-b_{i}$ columns. (Note that the first processor does not touch the first $b_{i} / 2$ columns, but rather stores them to be updated in the next step.) This distribution also implies that between steps $i$ and $i+1$, the $Q$ matrix must be shifted to maintain the relationship between the ownership of rows of the band and the columns of $Q$. To simplify the presentation, we 
assume that on each step $i, Q$ is padded with $b_{i}$ zero columns, and that the first processor in each row always sends its rightmost $C$ columns; under these assumptions, each processor always sends/receives $C$-by- $C$ blocks of $Q$, avoiding fringe cases for the first and last processors (within each processor row).

We introduce four kernels_create_bulges_update, pass_bulges_update, create_and_clear_bulges_update, and clear_bulges_update-which apply the right orthogonal updates (as sets of Householder transformations) from the corresponding band reduction kernels to the local blocks of $Q$.

Again, we do not analyze computing the eigendecomposition of $T$, but we assume that this step terminates with $Q_{2}$ distributed across the processor grid with each processor owning a $C$-by- $C$ block of $Q_{2}$. We then compute $Q_{1} Q_{2}$ using matrix-matrix multiplication.

In the following complexity analysis, we count only the additional work and communication done for the orthogonal updates. To obtain the results for CA-SBR in Table VI, we simply add the band reduction counts (Section 6.1.2), substituting $\sqrt{p}$ for $p$ (since now we run the band reduction redundantly). Then we add the counts of multiplying $Q_{1} Q_{2}$ with Cannon's algorithm [Cannon 1969], which requires $2 n^{3} / p$ flops, $O\left(n^{2} / \sqrt{p}\right)$ words moved, and $O(\sqrt{p})$ messages. These are all lower-order terms, due to the logarithmic factors in the other counts.

Flop Count. As argued in Section 6.1.2, there are at most $\omega_{i} \ell_{i}$ bulges chased in the pass_bulges, clear_bulges, and create_and_clear_bulges kernels, and at most $2 \omega_{i} \ell_{i}$ bulges chased in the create_bulges kernel. Since the number of Householder entries in each bulge chase is $c_{i} d_{i}=b_{i}^{2} / 4$, from Lemma 5.2, applying the updates from one kernel invocation to $n / \sqrt{p}$ rows of the $Q$ matrix requires at most

$$
4 \cdot \frac{b_{i}^{2}}{4} \cdot \frac{n}{\sqrt{p}} \cdot \omega_{i} \ell_{i}=\frac{2 n^{3}}{3 p^{3 / 2}}=O\left(\frac{n^{3}}{p^{3 / 2}}\right)
$$

flops (and up to 2 times more for create_bulges).

Following the analysis in Section 6.1.2, we can upper bound the additional arithmetic performed along the critical path by

$$
O\left(\frac{n^{3}}{p}\right)+\sum_{i=1}^{\log b} O\left(\frac{n^{3}}{p}\right)=O\left(\frac{n^{3} \log b}{p}\right)
$$

flops. The flop counts of the band reduction and multiplication $Q_{1} Q_{2}$ are lower-order terms.

Word Count. The communication costs of the orthogonal updates are also analogous to band reduction. As shown in Algorithm 4, for every message sent/received containing a block of $A$, there is a second message containing a block of $Q$. (The additional message every step to shift the block row of $Q$ amounts to a lower-order term.) However, while the size of the $A$ messages decreases with the bandwidth, the size of the $Q$ messages remains the same $\left(n^{2} / p\right.$ words). The additional word count, following the analysis in Section 6.1.2, is bounded by

$$
O\left(\frac{n^{2}}{\sqrt{p}}\right)+\sum_{i=1}^{\log b} O\left(\frac{n^{2}}{\sqrt{p}}\right)=O\left(\frac{n^{2} \log b}{\sqrt{p}}\right)
$$

words moved. Again, the word counts of the band reduction and multiplication $Q_{1} Q_{2}$ are lower-order terms. 


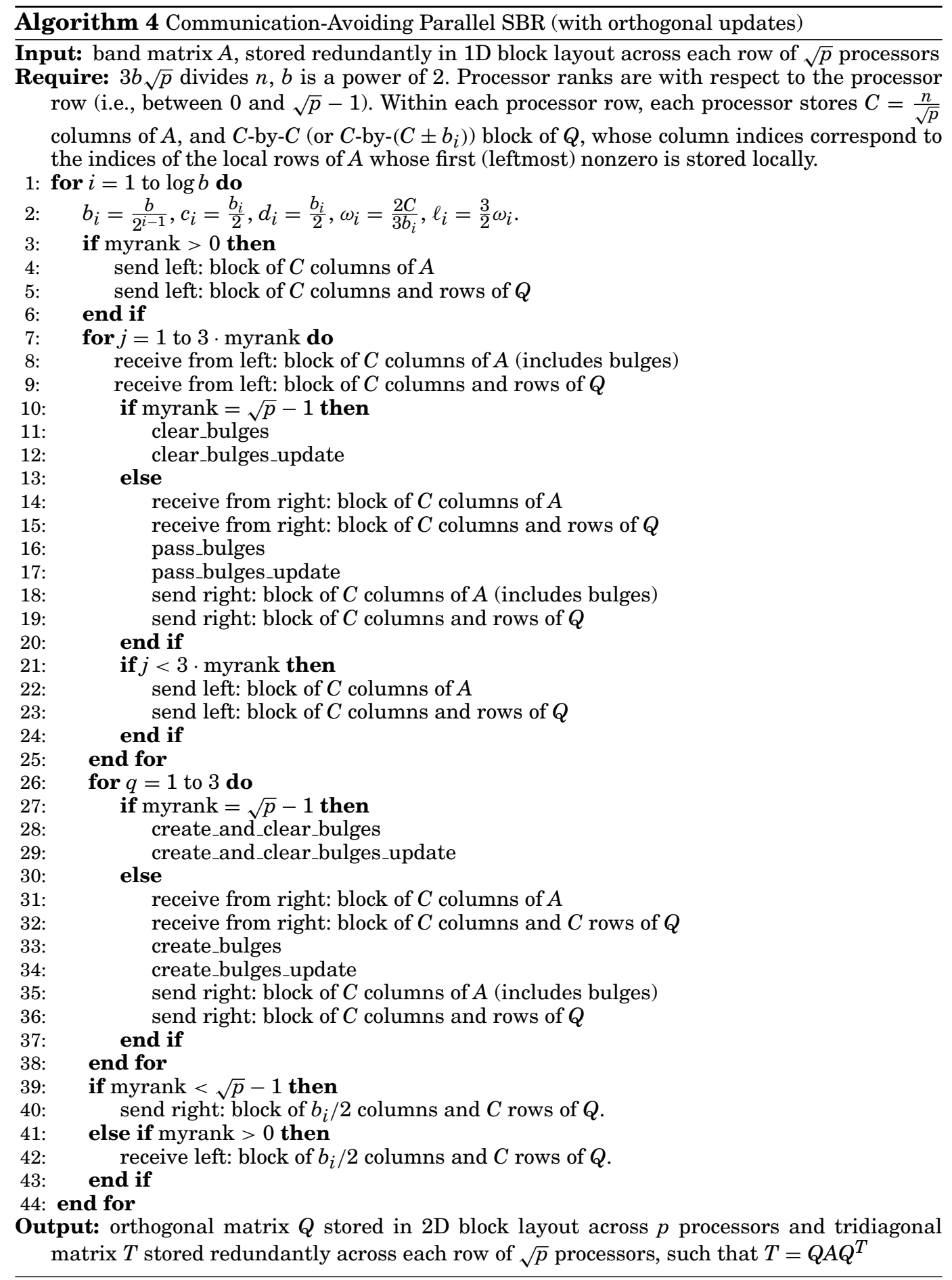


Message Count. The additional message count is the same as that for the band reduction (see Section 6.1.2) plus the shift (a lower-order term), that is, $O(\sqrt{p} \log b)$ messages. In the more restrictive case $\sqrt{p} \ll n /(b \log b)$, this is an asymptotic improvement compared to Lang's algorithm for just the back-transformation phase; considering also the counts of the band reduction, we always have an asymptotic improvement.

\section{CONCLUSIONS}

In theory, both band reduction and dense matrix-matrix multiplication have $O(n)$ possible data reuse in the sequential case, given by the ratio of total flops to size of inputs and outputs. When the problem does not fit in fast memory (of size $M$ words), matrix multiplication can attain only $O(\sqrt{M})$ data reuse [Hong and Kung 1981], while our CA-SBR algorithm achieves $O(M / b)$ reuse, provided $b \leq \sqrt{M} / 3$. This constraint on $b$ also ensures that the reuse is always asymptotically at least as large as that of matrix multiplication, and when $b \ll \sqrt{M}$, we can actually attain much better reuse.

Indeed, improved data reuse often translates to better performance. In Ballard et al. [2012], we observed that using the techniques of reducing communication (even at the expense of some extra arithmetic), as well as a framework that automatically tuned the algorithmic parameters, led to speedups on sequential and shared-memory parallel machines. We believe that these benefits will extend to the distributed-memory case, particularly when performance is latency-bound.

The performance results in Ballard et al. [2012] focused on the case of computing eigenvalues only and did not study the costs of the back-transformation phase, when eigenvectors are also computed. (Recall that the flop count of the band reduction phase varies by no more than $50 \%$ for different numbers of steps.) As we have seen, the costs of the back-transformation, which dominate those of the band reduction, increase with the number of steps. Thus, there exists an important trade-off between reducing communication in the band reduction phase and the resulting increased costs in the backtransformation phase. As mentioned earlier, when computing partial eigensystems, the costs of the back-transformation can be reduced to be proportional to the number of eigenvectors desired, improving this trade-off.

We also do not give algorithms or complexity analysis for taking more than 1 and less than $\log b$ steps and using the technique of chasing multiple bulges. Indeed, we fixed many parameters in this work with the sole intention of simplifying the theoretical analysis. In practice, parameters such as the number of steps and the number of bulges chased at a time should be autotuned for the target architecture to navigate the tradeoffs mentioned earlier.

We did not discuss composing our parallel and sequential algorithms in this work. Observing that the local computations in the parallel algorithms can be viewed as (portions of) the sequential algorithm, with a little care to deal with the ends of the band, one can apply our sequential analysis to model sequential communication within the parallel algorithms. One interesting tuning parameter that arises when composing the algorithms is the ability to implement the local (large) bulge chases by breaking them into more (smaller) bulge chases, now blocking for the local memory hierarchy. We also did not consider shared-memory parallelism in this work, but showed in a previous paper [Ballard et al. 2012] that our sequential algorithm, in the case of computing eigenvalues only, is easily parallelized in shared-memory settings. We claim that, in the case of computing both eigenvalues and eigenvectors (in shared memory), a similar pipelining strategy could be used during the band-reduction phase for updating the orthogonal matrix $Q$; there are no additional complications for parallelizing the backtransformation phase, because we implement it with matrix multiplication. Thus, we 
believe that the approaches in this article can be readily extended to hybrid-parallel implementations.

Recall our application of two-step tridiagonalization for the symmetric eigenproblem. The first step (full-to-banded) and its corresponding back-transformation phase, can be performed efficiently [Ballard et al. 2011a; Luszczek et al. 2011]. Combined with the approaches here for the second step (and an efficient tridiagonal eigensolver), we have sequential and parallel algorithms for the symmetric eigenproblem that attain the communication lower bounds for dense linear algebra in [Ballard et al. 2011b] up to $O(\log b)$ factors: in serial, $\Omega\left(n^{3} / \sqrt{M}\right)$ words moved and $\Omega\left(n^{3} / M^{3 / 2}\right)$ messages, in parallel (if minimal memory is used), $\Omega\left(n^{2} / \sqrt{p}\right)$ words moved and $\Omega(\sqrt{p})$ messages. Even though these lower bounds formally apply to only the first step, they are still valid lower bounds for any algorithm that performs this step.

As opposed to the SBR framework and other related works, our algorithms or analyses may not apply if the starting matrix is banded with bandwidth exceeding the constraints in Sections 5 and 6 . While one could always treat the starting band matrix as full, we then cannot guarantee the asymptotic communication optimality of the algorithms in Section 3, unless the starting bandwidth is within a constant factor of the matrix dimension. However, the blocking approaches in Section 3 can be easily extended to any bulge-chasing context, not just the special case of the full-to-band step. In the sequential case, we claim that, for any starting bandwidth, we can reduce to tridiagonal form with communication costs that attain (or beat) the aforementioned bounds. It is open whether the same approach can be as efficient in the parallel case, due to the complication of parallelizing individual bulge chases.

Finally, we reiterate that our approaches generalize to the tridiagonalization of Hermitian matrices, to the bidiagonalization or Hessenberg reduction of general rectangular matrices, or to any algorithm which reduces the upper and/or lower bandwidth by bulge-chasing (using two-sided orthogonal transformations). In the cases of bidiagonalization and Hermitian tridiagonalization, the analysis is identical up to constant factors. When symmetry need not be maintained, there is more flexibility in creating and chasing bulges both above and below the diagonal, and similar issues to those described in Section 4 regarding fill-in arise (e.g., from overlapping bulges).

\section{REFERENCES}

Aggarwal, A. and Vitter, J. 1988. The input/output complexity of sorting and related problems. Commun. ACM 31, 9, 1116-1127.

Agullo, E., Dongarra, J., Hadri, B., Kurzak, J., Langou, J., Langou, J., Ltaief, H., Luszczek, P., and Yarkhan, A. 2009. PLASMA Users' Guide. http://icl.cs.utk.edu/plasma/.

Anderson, E., Bai, Z., Bischof, C., et al. 1992. LAPACK Users' Guide. SIAM, Philadelphia, PA.

Auckenthaler, T. 2012. Highly scalable eigensolvers for petaflop applications. Ph.D. thesis, Fakultät für Informatik, Technische Universität München.

Auckenthaler, T., Blum, V., Bungartz, H.-J., Huckle, T., Johanni, R., Krämer, L., Lang, B., Lederer, H., and Willems, P. 2011a. Parallel solution of partial symmetric eigenvalue problems from electronic structure calculations. Parallel Comput. 37, 12, 783-794.

Auckenthaler, T., Bungartz, H.-J., Huckle, T., Krämer, L., Lang, B., Lederer, H., and Willems, P. 2011 b. Developing algorithms and software for the parallel solution of the symmetric eigenvalue problem. J. Computat. Sci. 2, 3, 272-278.

Ballard, G., Demmel, J., and Dumitriu, I. 2011a. Communication-optimal parallel and sequential eigenvalue and singular value algorithms. EECS Tech. Rep. EECS-2011-14, University of California, Berkeley.

Ballard, G., Demmel, J., Grigori, L., Jacquelin, M., Nguyen, H., and Solomonik, E. 2013a. Reconstructing householder vectors from tall-skinny QR. Tech. Rep. UCB/EECS-2013-175, EECS Department, University of California, Berkeley.

Ballard, G., Demmel, J., Holtz, O., and Schwartz, O. 2011b. Minimizing communication in numerical linear algebra. SIAM J. Matrix Anal. Appl. 32, 3, 866-901. 
Ballard, G., Demmel, J., and Knight, N. 2012. Communication avoiding successive band reduction. In Proceedings of the 17th ACM SIGPLAN Symposium on Principles and Practice of Parallel Programming (PPoPP'12). ACM, New York, 35-44.

Ballard, G., Demmel, J., Lipshitz, B., Schwartz, O., and Toledo, S. 2013b. Communication efficient Gaussian elimination with partial pivoting using a shape morphing data layout. In Proceedings of the 25th ACM Symposium on Parallelism in Algorithms and Architectures (SPAA'13). ACM, 232-240.

Barth, W., Martin, R., and Wilkinson, J. 1967. Calculation of the eigenvalues of a symmetric tridiagonal matrix by the method of bisection. Numerische Mathematik 9, 5, 386-393.

Bientinesi, P., Igual, F., Kressner, D., and Quintana-Ort, E. 2010. Reduction to condensed forms for symmetric eigenvalue problems on multi-core architectures. In Parallel Processing and Applied Mathematics, R. Wyrzykowski, J. Dongarra, K. Karczewski, and J. Wasniewski, Eds., Lecture Notes in Computer Science, vol. 6067, Springer, 387-395.

Bischof, C., Lang, B., and Sun, X. 1994. Parallel tridiagonalization through two-step band reduction. In Proceedings of the Conference on Scalable High-Performance Computing. IEEE, 23-27.

Bischof, C., Lang, B., and Sun, X. 2000a. Algorithm 807: The SBR Toolbox: Software for successive band reduction. ACM Trans. Math. Softw. 26, 4, 602-616.

Bischof, C., Lang, B., and Sun, X. 2000b. A framework for symmetric band reduction. ACM Trans. Math. Softw. 26, 4, 581-601.

Bischof, C., Marques, M., and Sun, X. 1993. Parallel bandreduction and tridiagonalization. In Proceedings of the 6th SIAM Conference on Parallel Processing for Scientific Computing. Vol. 1, SIAM, 383-390.

Bischof, C. and Sun, X. 1992. A framework for symmetric band reduction and tridiagonalization. Tech. Rep. MCS-P298-0392, Argonne National Laboratory.

Blackford, L. S., Choi, J., Cleary, A., et al. 1997. ScaLAPACK Users' Guide. SIAM, Philadelphia, PA. http://www.netlib.org/scalapack/.

Bowdler, H., Martin, R., Reinsch, C., and Wilkinson, J. 1968. The QR and QL algorithms for symmetric matrices. Numerische Mathematik 11, 4, 293-306.

Braman, K., Byers, R., and Mathias, R. 2002. The multishift QR algorithm. part i: Maintaining wellfocused shifts and level 3 performance. SIAM J. Matrix Anal. Appl. 23, 4, 929-947.

Bruck, J., Ho, C.-T., Kipnis, S., and Weathersby, D. 1994. Efficient algorithms for all-to-all communications in multi-port message-passing systems. In Proceedings of the 6th Annual ACM Symposium on Parallel Algorithms and Architectures (SPAA'94). ACM, New York, 298-309.

Cannon, L. 1969. A cellular computer to implement the Kalman filter algorithm. Ph.D. thesis, Montana State University, Bozeman, MT.

Cuppen, J. 1980. A divide and conquer method for the symmetric tridiagonal eigenproblem. Numerische Mathematik 36, 2, 177-195.

Demmel, J., Grigori, L., Hoemmen, M., and Langou, J. 2012. Communication-optimal parallel and sequential QR and LU factorizations. SIAM J. Sci. Comput. 34, 1, A206-A239.

Demmel, J., Marques, O., Parlett, B., and Vömel, C. 2008. Performance and accuracy of LAPACK's symmetric tridiagonal eigensolvers. SIAM J. Sci. Comput. 30, 3, 1508-1526.

Dhillon, I. S. and Parlett, B. N. 2004. Multiple representations to compute orthogonal eigenvectors of symmetric tridiagonal matrices. Linear Algebra Appl. 387, 1-28.

Dongarra, J. J., Sorensen, D. C., and Hammarling, S. J. 1989. Block reduction of matrices to condensed forms for eigenvalue computations. J. Comput. Appl. Math. 27, 1-2, 215-227.

Fuller, S. and Millett, L., Eds. 2011. The Future of Computing Performance: Game Over or Next Level? National Academies Press, Washington, D.C.

Gansterer, W. N., Kvasnicka, D. F., and Ueberhuber, C. W. 1999. Multi-sweep algorithms for the symmetric eigenproblem. In Vector and Parallel Processing, V. Hernandez, J. Palma, and J. J. Dongarra, Eds., Lecture Notes in Computer Science, vol. 1573, Springer, 20-28.

Granat, R., Kågström, B., and Kressner, D. 2010. A novel parallel QR algorithm for hybrid distributed memory hpc systems. SIAM J. Sci. Comput. 32, 4, 2345-2378.

Grosser, B. and Lang, B. 1999. Efficient parallel reduction to bidiagonal form. Parallel Comput. 25, 8, 969-986.

$\mathrm{Gu}, \mathrm{M}$. and Eisenstat, S. 1992. A stable algorithm for the rank-1 modification of the symmetric eigenproblem. Tech. Rep. YALEU/DCS/RR-916, Yale University.

Haidar, A., Kurzak, J., and Luszczek, P. 2013a. An improved parallel singular value algorithm and its implementation for multicore hardware. In Proceedings the International Conference for High Performance Computing, Networking, Storage and Analysis. ACM, New York, 90:1-90:12. 
Haidar, A., Ltaief, H., and Dongarra, J. 2011. Parallel reduction to condensed forms for symmetric eigenvalue problems using aggregated fine-grained and memory-aware kernels. In Proceedings of the International Conference for High Performance Computing, Networking, Storage and Analysis. ACM, 8.

Haidar, A., Ltaief, H., and Dongarra, J. 2012a. Toward a high performance tile divide and conquer algorithm for the dense symmetric eigenvalue problem. SIAM J. Sci. Comput. 34, 6, C249-C274.

Haidar, A., Ltaief, H., Luszczek, P., and Dongarra, J. 2012b. A comprehensive study of task coalescing for selecting parallelism granularity in a two-stage bidiagonal reduction. In Proceedings of the IEEE 26th International Parallel \& Distributed Processing Symposium. IEEE, 25-35.

Haidar, A., Tomov, S., Dongarra, J., Solcà, R., and Schulthess, T. 2013b. A novel hybrid CPU-GPU generalized eigensolver for electronic structure calculations based on fine-grained memory aware tasks. Int. J. High Perform. Comput. Appl.

Hong, J. and Kung, H. 1981. I/O complexity: The red-blue pebble game. In Proceedings of the 13th Annual ACM Symposium on Theory of Computing (STOC'81). ACM, New York, 326-333.

Howell, G. W., Demmel, J. W., Fulton, C. T., Hammarling, S., and Marmol, K. 2008. Cache efficient bidiagonalization using BLAS 2.5 operators. ACM Trans. Math. Softw. 34, 3, 14:1-14:33.

Karlsson, L. and Kågström, B. 2011. Parallel two-stage reduction to Hessenberg form using dynamic scheduling on shared-memory architectures. Parallel Comput. 37, 12, 771-782.

Kaufman, L. 1984. Banded eigenvalue solvers on vector machines. ACM Trans. Math. Softw. 10, 73-86.

Kaufman, L. 2000. Band reduction algorithms revisited. ACM Trans. Math. Softw. 26, 551-567.

Lang, B. 1991. Parallele reduktion symmetrischer bandmatrizen auf tridiagonalgestalt. Ph.D. thesis, Fakultät für Informatik, Technische Universität München.

Lang, B. 1993. A parallel algorithm for reducing symmetric banded matrices to tridiagonal form. SIAM J. Sci. Comput. 14, 6, 1320-1338.

Lang, B. 1996. Parallel reduction of banded matrices to bidiagonal form. Parallel Comput. 22, 1, 1-18.

Lang, B. 1999. Efficient eigenvalue and singular value computations on shared memory machines. Parallel Comput. 25, 7, 845-860.

Ltaief, H., Luszczek, P., and Dongarra, J. 2013. High-performance bidiagonal reduction using tile algorithms on homogeneous multicore architectures. ACM Trans. Math. Softw. 39, 3, 16:1-16:22.

Luszczek, P., Ltaief, H., and Dongarra, J. 2011. Two-stage tridiagonal reduction for dense symmetric matrices using tile algorithms on multicore architectures. In Proceedings of the IEEE International Parallel \& Distributed Processing Symposium (IPDPS'11). IEEE, 944-955.

Murata, K. and Horikoshi, K. 1975. A new method for the tridiagonalization of the symmetric band matrix. Inf. Process. Japan 15, 108-112.

Rajamanickam, S. 2009. Efficient algorithms for sparse singular value decomposition. Ph.D. thesis, University of Florida.

Rutishauser, H. 1963. On Jacobi rotation patterns. In Proceedings of Symposia in Applied Mathematics. Vol. 15, AMS, 219-239.

Schreiber, R. and Van Loan, C. 1989. A storage-efficient wy representation for products of householder transformations. SIAM J. Sci. Stat. Comput. 10, 1, 53-57.

Schwarz, H. 1963. Algorithm 183: Reduction of a symmetric bandmatrix to triple diagonal form. Commun. ACM 6, 6, 315-316.

Schwarz, H. 1968. Tridiagonalization of a symmetric band matrix. Numerische Mathematik 12, 231-241.

Smith, C., Hendrickson, B., and Jessup, E. 1994. A parallel algorithm for householder tridiagonalization. In Proceedings of the 5th SIAM Conference on Applied Linear Algebra. 361-365.

Van Zee, F., Van De Geijn, R., and Quintana-Orti, G. 20134. Restructuring the QR algorithm for performance. ACM Trans. Math. Softw. 40, 3.

Wilkinson, J. 1962. Householder's method for symmetric matrices. Numerische Mathematik 4, 1, 354-361.

Yamazaki, I., Dong, T., Tomov, S., and Dongarra, J. 2013. Tridiagonalization of a symmetric dense matrix on a GPU cluster. In Proceedings of the 27th International Parallel and Distributed Processing Symposium Workshops PhD Forum. 1070-1079.

Received April 2013; revised July 2014; accepted July 2014 